\title{
Information and disturbance in operational probabilistic theories
}

\author{
Giacomo Mauro D'Ariano, Paolo Perinotti, and Alessandro Tosini \\ QUIT group, Physics Dept., Pavia University, and INFN Sezione di Pavia, via Bassi 6, 27100 Pavia, Italy
}

Any measurement is intended to provide information on a system, namely knowledge about its state. However, we learn from quantum theory that it is generally impossible to extract information without disturbing the state of the system or its correlations with other systems. In this paper we address the issue of the interplay between information and disturbance for a general operational probabilistic theory. The traditional notion of disturbance considers the fate of the system state after the measurement. However, the fact that the system state is left untouched ensures that also correlations are preserved only in the presence of local discriminability. Here we provide the definition of disturbance that is appropriate for a general theory. Moreover, since in a theory without causality information can be gathered also on the effect, we generalise the notion of noinformation test. We then prove an equivalent condition for no-information without disturbance-atomicity of the identity namely the impossibility of achieving the trivial evolution - the identity - as the coarse-graining of a set of non trivial ones. We prove a general theorem showing that information that can be retrieved without disturbance corresponds to perfectly repeatable and discriminating tests. Based on this, we prove a structure theorem for operational probabilistic theories, showing that the set of states of any system decomposes as a direct sum of perfectly discriminable sets, and such decomposition is preserved under system composition. As a consequence, a theory is such that any information can be extracted without dis-

Giacomo Mauro D’Ariano: dariano@unipv.it

Paolo Perinotti: paolo.perinotti@unipv.it

Alessandro Tosini: alessandro.tosini@unipv.it turbance only if all its systems are classical. Finally, we show via concrete examples that no-information without disturbance is independent of both local discriminability and purification.

\section{1 introduction}

The possibility that gathering information on a physical system may affect the state of the system itself was introduced by Heisenberg in his famous gedanken experiment [1], which became the first paradigm of quantum mechanics. The issue raised by Heisenberg spawned a vaste literature up to present days (see [2,3] as recent reviews), with a variety of quantifications of "information" and "disturbance" and corresponding tradeoff relations $[4,5,6,7]$. All these results are quantitative accounts of a core issue in quantum theory, the no-information without disturbance theorem $[8,9]$. The proofs of the theorem rely on the mathematical structure of quantum theory, and thus do not emphasise the logical relation between no-information without disturbance and other quantum features, such as local discriminability (the possibility of discriminating multipartite states via only local measurements) or purification (every mixed state can be obtained as the marginal state of a pure state).

The framework here used for exploring the relation between information and disturbance is that of operational probabilistic theories (OPTs) [10, $11,9]$. In this setting a rigorous formulation of the notions of system, process, and their compositions is given, which constitutes the grammar for the probabilistic description of an experiment. Quantum theory and classical theory are two instances of OPTs.

For some probabilistic theories which can be reframed as OPTs, the definitions of information and disturbance have been investigated in the presence of local discriminability, purifica- 
tion, and causality $[12,13,14,15]$. For OPTs satisfying those three axioms the no-information without disturbance theorem has been proved in Refs. [10, 9]. In the present paper we point out a weakness in the existing notion of disturbance, which is ubiquitous in all past approaches. Indeed, the conventional definition of disturbance asserts that an experiment does not disturb the system if and only if its overall effect is to leave unchanged the states of the system, disregarding the effects of the experiment on the environment. Whilst this captures the meaning of disturbance within quantum theory, we cannot consistently apply the same notion in theories that violate local discriminability. A significative case is that of the Fermionic theory $[16,17,18]$ where, due to the parity superselection rule, an operation that does not disturb a bunch of Fermionic systems still could affect their correlations with other systems. This issue can be cured asking a non-disturbing experiment to preserve not only the system state, but also its purifications [10, 9]. This extension of the notion of disturbance is general enough to capture the operational meaning of disturbance for Fermionic systems, however, it is still unsatisfactory, since it cannot be used to describe disturbance in models that do not enjoy purification, e. g. classical information theory.

Here we will define non-disturbing operations only by referring to the OPT framework, thus providing a notion that holds also for theories that do not satisfy local discriminability, purification, or causality, and even for theories whose sets of states are not convex. Given a system, and an operation on it, the fate of any possible dilation of the states of the system after the operation is taken into account, where by dilation we mean any state of a larger system whose marginal is the dilated state ${ }^{1}$. Moreover, due to the lack of causality, effects and states must be treated on the same footing, and we extend the notion of information also encompassing the information about the output. We prove then a necessary and sufficient condition for a theory to satisfy no-information without disturbance. The condition is the impossibility of realizing the identity transformation as a nontrivial coarse-graining of a set of operations. Technically speaking the

\footnotetext{
${ }^{1}$ We remind that for non-causal theories the marginal is not unique, hence more generally, we require that one of the marginals is the given state.
}

above condition amounts to atomicity of the identity. Finally, since a theory might satisfy noinformation without disturbance only when restricted to some collections of preparations and measurements, we will provide a weaker necessary and sufficient condition for this case.

Similarly to the Heisenberg uncertainty relations, no-information without disturbance has been considered as a characteristic quantum trait. Instead, as we will see here, this feature can be exhibited in the absence of most of the principles of quantum theory [9], and it is ubiquitous among OPTs. Moreover, the most general case is that of an OPT where some information can be extracted without disturbance, in which case this information has all the features of a classical one. On the other hand, the only kind of systems that allow for extracting any information without disturbance are classical systems. This observation provides an alternative way of characterising classical systems with respect to Ref. [19].

In Section 2 we review the framework of operational probabilistic theories and some relevant features that characterize quantum theory within this scenario. In Section 3, after introducing the definition of information and disturbance, we present the main results of this paper: i) the atomicity of the identity evolution as a necessary and sufficient condition for no-information without disturbance; ii) other equivalent necessary and sufficient conditions in terms of properties of reversible evolutions of the theory; iii) we prove a structure theorem for theories where some information can be extracted without disturbance; iv) we prove that the information that can be extracted without disturbance is "classical", in the sense that its measurement is a repeatable reading of shareable information; v) finally we prove that a theory in which any information can be extracted without disturbance is a theory where all systems are classical. In Section 4 we generalize the notion of equality upon input to general OPTs, including the cases in which local discriminability does not hold. Moreover, dealing also with non-causal theories, where states and effects must be considered on the same footing, we introduce the notion of equality upon input and upon output. This notion can be used when only a subset of the preparations and of the measurements are accessible, e.g. in resource theories [20, 21]. As a first application we generalize the notion of 
information and disturbance to the upon input and upon output scenario, providing a characterization of the no-information without disturbance also in this case. In Section 5 we deepen the relation between no-information without disturbance and other characteristic properties of quantum theory. We show that no-information without disturbance can be satisfied independently of purification and local discriminability, providing counterexamples based on some of the conditions mentioned above and other conditions proved in this section. We end with the conclusions in Section 6 .

\section{The Framework}

In this section we review the framework of $o p$ erational probabilistic theories (OPT) (we refer to $[10,9,11]$ for further details).

The primitives of an operational theory are the notions of test, event, and system. A test $\left\{\mathcal{A}_{i}\right\}_{i \in \mathrm{X}}$ is the collection of events $\mathcal{A}_{i}$, where $i$ labels the element of the outcome space X. In the quantum case $\mathcal{A}_{i}$ is the $i$ th quantum operation of the quantum instrument $\left\{\mathcal{A}_{i}\right\}_{i \in \mathrm{X}}$. The notion of test bridges the experiment with the theory, with $i \in \mathrm{X}$ denoting the objective outcome, and $\mathcal{A}_{i}$ the mathematical description of the corresponding event. The notion of system, here denoted by capital Roman letters A, B, ..., rules connections of tests. An input and an output label are associated to any test (event). We represent a test $\mathcal{A}_{\mathrm{X}}:=\left\{\mathcal{A}_{i}\right\}_{i \in \mathrm{X}}$ and its building events $\mathcal{A}_{i}$ by the diagrams

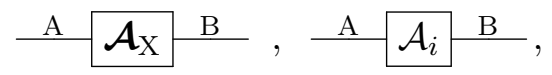

respectively, with the rule that an output wire can be connected only to an input wire with the same label. Thus, given two tests $\mathcal{A}_{\mathrm{X}}$ and $\boldsymbol{\mathcal { B }}_{\mathrm{Y}}$ we can define their sequential composition $(\mathcal{B A})_{\mathrm{X} \times \mathrm{Y}}$ as the collection of events

$$
\mathrm{A} \mathcal{B}_{j} \mathcal{A}_{i} \stackrel{\mathrm{C}}{=}=\stackrel{\mathrm{A}}{\mathcal{A}_{i}} \mathrm{~B} \mathcal{B}_{j} \mathrm{C},
$$

for $i \in \mathrm{X}$ and $j \in \mathrm{Y}$. A singleton test is a test containing a single event. We call such an event deterministic. For every system A there exists a unique singleton test $\left\{\mathcal{I}_{\mathrm{A}}\right\}$ such that $\mathcal{I}_{\mathrm{B}} \mathcal{A}=\mathcal{A I}_{\mathrm{A}}=\mathcal{A}$ for every event $\mathcal{A}$ with input $\mathrm{A}$ and output $\mathrm{B}$, and we call $\mathcal{I}_{\mathrm{A}}$ identity of system A. Besides sequential compositions of tests and events, a theory is specified by the rule for composing them in parallel. For every couple of systems (A, B) we can form the composite system $\mathrm{C}:=\mathrm{AB}$, on which we can perform tests $(\mathcal{C} \otimes \mathcal{D})_{\mathrm{X} \times \mathrm{Y}}$ with events $\mathcal{C}_{i} \otimes \mathcal{D}_{j}$ in parallel composition represented as follows

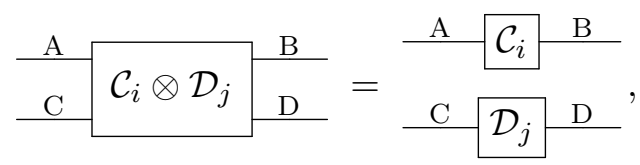

and satisfying the condition $\left(\mathcal{E}_{h} \otimes \mathcal{F}_{k}\right)\left(\mathcal{C}_{i} \otimes \mathcal{D}_{j}\right)=$ $\left(\mathcal{E}_{h} \mathcal{C}_{i}\right) \otimes\left(\mathcal{F}_{k} \mathcal{D}_{j}\right)$. Notice that we use the tensor product symbol $\otimes$ for the parallel composition rule. Actually, for the quantum and the classical OPT the parallel composition is the usual tensor product of linear maps. However, for a general OPT, the parallel composition may not coincide with a tensor product.

There exists a special system type I, the trivial system, such that $\mathrm{AI}=\mathrm{IA}=\mathrm{A}$ for every system A. The tests with input system I and output A are called preparation tests of $\mathrm{A}$, while the tests with input system A and output I are called $o b$ servation tests of A. Preparation events of A are graphically denoted as boxes without the input wire $\rho$ A (or in formula as round kets $\mid \rho)_{\mathrm{A}}$ ), and the observation events by boxes with no output wire $\frac{\mathrm{A}}{c}$ (in formula round bras $\left(\left.c\right|_{\mathrm{A}}\right)$. For example, one can have events of the following kind

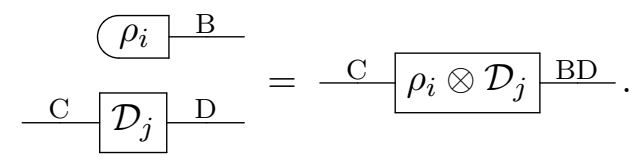

We will always use the Greek letters to denote preparation tests $\left\{\rho_{i}\right\}_{i \in \mathrm{X}}$ and Latin letters to denote observation tests $\left\{c_{j}\right\}_{j \in \mathrm{X}}$ (we will not specify the system when it is clear from the context).

An arbitrary test obtained by parallel and sequential composition of box diagrams is called circuit. A circuit is closed if its overall input and output systems are trivial: it starts with a preparation test and ends with an observation test. An operational probabilistic theory (OPT) is an operational theory where any closed circuit of tests corresponds to a probability distribution for the joint test. Compound tests from the trivial system to itself are independent, both for sequential and parallel composition, namely their joint probability distribution is given by the product of the respective joint probability distributions. For example the application of an observation event $c_{i}$ 
after the preparation event $\rho_{j}$ corresponds to the closed circuit $\left(c_{i} \mid \rho_{j}\right)_{\mathrm{A}}$ and denotes the probability of the outcome $(i, j)$ of the observation test $\boldsymbol{c}_{\mathrm{X}}$ after the preparation test $\boldsymbol{\rho}_{\mathrm{Y}}$ of system A, i.e.

$$
\rho_{j} \mathrm{~A}, c_{i}:=\operatorname{Pr}\left[i, j \mid \rho_{\mathrm{Y}} \mathrm{A}, \boldsymbol{c}_{\mathrm{X}}\right] .
$$

For a more complex example, consider the test

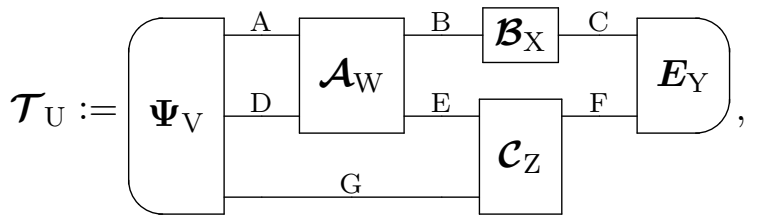

with $\mathrm{U}=\mathrm{V} \times \mathrm{W} \times \mathrm{X} \times \mathrm{Y} \times \mathrm{Z}$. Then we define

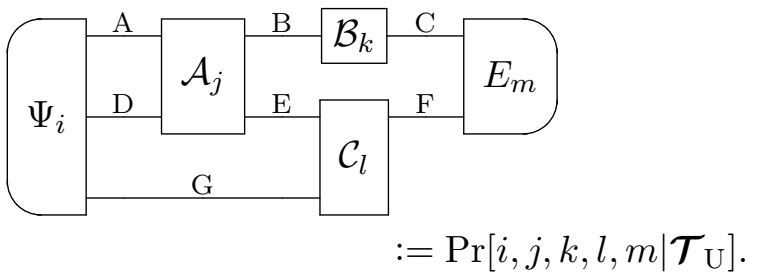

In the following, we will omit the parametric dependence on the circuit if the latter is clear from the context.

Summarising: by a closed circuit made of events we denote their joint probability upon the connection specified by the circuit graph, with nodes being the test boxes, and links being the system wires.

Given a system A of a probabilistic theory we can quotient the set of preparation events of $\mathrm{A}$ by the equivalence relation $\left.\mid \rho)_{\mathrm{A}} \sim \mid \sigma\right)_{\mathrm{A}} \Leftrightarrow(c \mid \rho)_{\mathrm{A}}=$ $(c \mid \sigma)_{\mathrm{A}}$ for every observation event $c$. Similarly we can quotient observation events. The equivalence classes of preparation events and observation events of $\mathrm{A}$ will be denoted by the same symbols as their elements $\mid \rho)_{\mathrm{A}}$ and $\left(\left.c\right|_{\mathrm{A}}\right.$, respectively, and will be called state and effect for system A.

For every system A, we will denote by $\mathrm{St}(\mathrm{A})$, $\operatorname{Eff}(\mathrm{A})$ the sets of states and effects, respectively. States and effects are real-valued functionals on each other, and can be naturally embedded in reciprocally dual real vector spaces, $\mathrm{St}_{\mathbb{R}}(\mathrm{A})$ and $\operatorname{Eff}_{\mathbb{R}}(\mathrm{A})$, whose dimension $\operatorname{dim}(\mathrm{A})$ is assumed to be finite.

In Appendix $\mathrm{A}$ it is proved that an event $\mathcal{A}$ with input system $\mathrm{A}$ and output system $\mathrm{B}$ induces a linear map from $\mathrm{St}_{\mathbb{R}}(\mathrm{AC})$ to $\mathrm{St}_{\mathbb{R}}(\mathrm{BC})$ for each ancillary system $\mathrm{C}$. The collection of all these maps is called transformation from A to B. More explicitly, given two transformations
$\mathcal{A}, \mathcal{A}^{\prime} \in \operatorname{Transf}(\mathrm{A}, \mathrm{B})$, one has $\mathcal{A}=\mathcal{A}^{\prime}$, if and only if

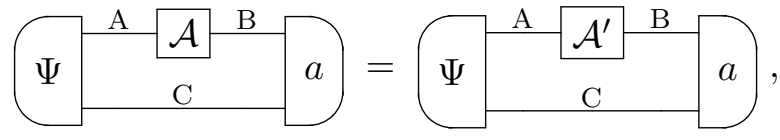

for every $\mathrm{C}$, every $\Psi \in \mathrm{St}(\mathrm{AC})$, and every $a \in$ $\operatorname{Eff}(\mathrm{BC})$, namely they give the same probabilities within every possible closed circuit. Notice that, using the fact that two states (effects) are equal if and only if they give the same probability when paired to every effect (state), the above condition amounts to state that $\mathcal{A}=\mathcal{A}^{\prime}$ if and only if

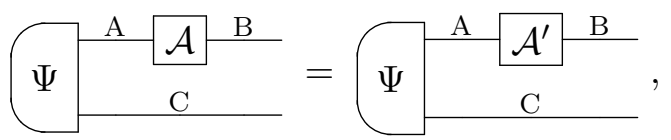

for every C, and every $\Psi \in \mathrm{St}(\mathrm{AC})$, or

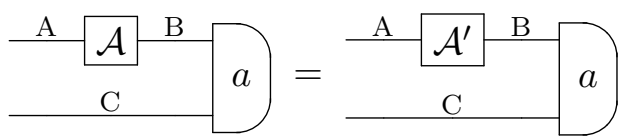

for every C, and every $a \in \operatorname{Eff}(\mathrm{BC})$.

In the following, the symbols $\mathcal{A}$ and $\mathrm{A} \mathcal{A}^{\mathrm{B}}$ will be used to represent the transformation corresponding to the event $\mathcal{A}$. The set of transformations from A to B will be denoted by $\operatorname{Transf}(\mathrm{A}, \mathrm{B})$, with linear span $\operatorname{Transf}_{\mathbb{R}}(\mathrm{A}, \mathrm{B})$. It is now obvious that a linear map $\mathcal{A} \in \operatorname{Transf}_{\mathbb{R}}(\mathrm{A}, \mathrm{B})$ is admissible if it locally preserves the set of states $\mathrm{St}(\mathrm{AC})$, namely $\mathcal{A} \otimes \mathcal{I}_{\mathrm{C}}(\mathrm{St}(\mathrm{AC})) \subseteq \mathrm{St}(\mathrm{BC})$, for every system C. In the following we will write $\mathcal{A} \mid \Psi)_{\mathrm{AC}}$ instead of $\left.\mathcal{A} \otimes \mathcal{I}_{\mathrm{C}} \mid \Psi\right)_{\mathrm{AC}}$, with $\Psi \in \mathrm{St}(\mathrm{AC})$ and $\mathcal{A} \in \operatorname{Transf(A,B)}$ when the domains are clear from the context.

An operational probabilistic theory is now defined as a collection of systems and transformations with the above rules for parallel and sequential composition and with a probability associated to any closed circuit ${ }^{2}$.

We introduce now the notions of refinement of an event and atomic event.

Definition 1 (Refinement of an event). A refinement of an event $\mathcal{C} \in \operatorname{Transf}(\mathrm{A}, \mathrm{B})$ is given by a collection of events $\left\{\mathcal{D}_{i}\right\}_{i \in \mathrm{X}}$ from $\mathrm{A}$ to $\mathrm{B}$, such that there exists a test $\left\{\mathcal{D}_{i}\right\}_{i \in \mathrm{Y}}$ with $X \subseteq Y$ and $\mathcal{C}=\sum_{i \in \mathrm{X}} \mathcal{D}_{i}$. We say that a refinement $\left\{\mathcal{D}_{i}\right\}_{i \in \mathrm{X}}$ of $\mathcal{C}$ is trivial if $\mathcal{D}_{i}=\lambda_{i} \mathcal{C}, \lambda_{i} \in[0,1]$, for every

${ }^{2}$ Notice that a more detailed account needs a categorytheoretical definition of parallel and sequential composition of systems (see Ref. [11]). 
$i \in \mathrm{X}$. Conversely, $\mathcal{C}$ is called the coarse-graining of the events $\left\{\mathcal{D}_{i}\right\}_{i \in \mathrm{X}}$, which we will also denote as $\mathcal{C}=\mathcal{D}_{\mathrm{X}}$.

In the following we will often refer to a refinement of $\mathcal{C}$ simply as $\mathcal{C}=\sum_{i \in \mathrm{X}} \mathcal{D}_{i}$, without specifying the test including the events $\mathcal{D}_{i}$.

Definition 2 (Refining event). Given two events $\mathcal{C}, \mathcal{D} \in \operatorname{Transf}(\mathrm{A}, \mathrm{B})$ we say that $\mathcal{D}$ refines $\mathcal{C}$, and write $\mathcal{D} \prec \mathcal{C}$, if there exist a refinement $\left\{\mathcal{D}_{i}\right\}_{i \in \mathrm{X}}$ of $\mathcal{C}$ such that $\mathcal{D} \in\left\{\mathcal{D}_{i}\right\}_{i \in \mathrm{X}}$.

Definition 3 (Non redundant test). We call a test $\left\{\mathcal{A}_{i}\right\}_{i \in \mathrm{X}}$ non redundant when for every pair $i, j \in \mathrm{X}$ one has $\mathcal{A}_{i} \neq \lambda \mathcal{A}_{j}$ for $\lambda>0$.

Notice that a test that is redundant can be interpreted as a non redundant test followed by a conditional coin tossing. As a consequence a redundant test always gives some spurious information, unrelated to the input state. From a redundant test one can achieve a maximal non redundant one by taking the test made of coarse grainings of all the sets of proportional elements.

Definition 4 (Refinement set). Given an event $\mathcal{C} \in \operatorname{Transf}(\mathrm{A}, \mathrm{B})$ we define its refinement set $\operatorname{Ref}_{\mathcal{C}}$ the set of all events that refine $\mathcal{C}$.

Definition 5 (Atomic and refinable events). An event $\mathcal{C}$ is atomic if it admits only of trivial refinements, namely $\mathcal{D} \prec \mathcal{C}$ implies $\mathcal{D}=\lambda \mathcal{C}$, $\lambda \in[0,1]$. An event is refinable if it is not atomic.

In the special case of states, the word pure is used as synonym of atomic, with a pure state describing an event that provides maximal knowledge about the system's preparation. This means that the knowledge provided by a pure state cannot be further refined. As usual a state that is not pure will be called mixed.

Another important relation between events is that of coexistence and the consequent notion of coexistent completion for a set o events.

Definition 6 (Coexistent events and coexistent completion). Two events $\mathcal{A}, \mathcal{B} \in \operatorname{Transf}(\mathrm{A}, \mathrm{B})$ are coexistent, and we write $\mathcal{A} \wedge \mathcal{B}$, if there exists a test $\left\{\mathcal{C}_{i}\right\}_{i \in \mathrm{X}} \subseteq \operatorname{Transf}(\mathrm{A}, \mathrm{B})$ such that $\mathcal{A}=\mathcal{C}_{\mathrm{Y}}$ and $\mathcal{B}=\mathcal{C}_{\mathrm{Z}}$, where $\mathrm{Y}, \mathrm{Z} \subseteq \mathrm{X}$. Given an event $\mathcal{A}$ we denote by $\widehat{\mathcal{A}}$ the set of all events coexistent with $\mathcal{A}$, and more generally, given a set of events $X$ its coexistent completion is defined as

$$
\widehat{X}=\{\mathcal{B} ; \mathcal{B} \wedge \mathcal{A}, \text { for some } \mathcal{A} \in X\} .
$$

We observe that in the present general OPT framework features that seem intuitive are not assumed, such as the convex completion of transformations. A remarkable example is that of "norestriction of preparation tests" hypothesis, consisting in the requirement that every collection of states that sum to a deterministic state is a preparation test. Similarly, we do not assume the no-restriction hypothesis for transformations, namely the requirement that every transformation that preserves the state set belongs to a test.

A fundamental definition for this manuscript is that that of dilation.

Definition 7 (Dilation). We say that $\Psi \in$ $\mathrm{St}(\mathrm{AB})$ is a dilation of $\rho \in \mathrm{St}(\mathrm{A})$ if

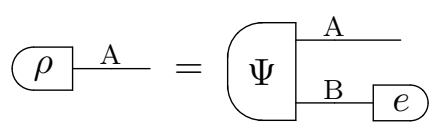

for some deterministic effect $e \in \mathrm{Eff}(\mathrm{B})$. Analogously, $c \in \operatorname{Eff}(\mathrm{AB})$ is a dilation of $a \in \operatorname{Eff}(\mathrm{A})$ if

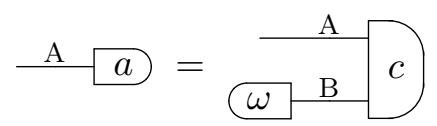

for some deterministic state $\omega \in \mathrm{St}(\mathrm{B})$. We denote by $D_{\rho}$ the set of all dilations of the state $\rho$. More generally, given a collection of states $S \subseteq \mathrm{St}(\mathrm{A})$ we define $D_{S}:=\bigcup_{\rho \in S} D_{\rho}$, with $D_{\mathrm{St}(\mathrm{A})}$ corresponding to the set of all states $\psi \in \mathrm{St}(\mathrm{AB})$ for every system $\mathrm{B}$. The same notation is used for the set of dilations of effects.

We remark that, given $\sigma \in S$, every state of the form $\sigma \otimes \rho$ belongs to $D_{S}$.

Notice that there are generally more than one deterministic effect for the same system, differently from quantum theory, where the partial trace over the Hilbert space of the system is the only way to discard it. Instead, given a state $\Psi \in \mathrm{St}(\mathrm{AB})$, in a theory with more deterministic effects for the same system B the marginal state of system A generally depends on the effect used to discard the system B. In the following we will call marginal of a state with deterministic effect $e$ the specific marginal obtained by applying the effect $e \in \operatorname{Eff}(\mathrm{B})$. Similarly, given an effect $c \in \operatorname{Eff}(\mathrm{AB})$ its marginal of system A depends on the choice of deterministic state on system $\mathrm{B}$ and we will call marginal of an effect with deterministic sate $\omega$ the specific marginal obtained by applying the deterministic state $\omega \in \mathrm{St}(\mathrm{B})$. 
Given a system A, in the dilations sets $D_{\mathrm{St}(\mathrm{A})}$ and $D_{\text {Eff(A) }}$, there could be states and effects with the following property.

Definition 8 (Faithful state and faithful effect). A state $\Psi \in \mathrm{St}(\mathrm{AC})$ is faithful for system $\mathrm{A}$ if given two transformations $\mathcal{A}, \mathcal{A}^{\prime} \in \operatorname{Transf}(\mathrm{A}, \mathrm{B})$, the condition $\left.\mathcal{A} \mid \Psi)_{\mathrm{AC}}=\mathcal{A}^{\prime} \mid \Psi\right)_{\mathrm{AC}}$ implies $\mathcal{A}=$ $\mathcal{A}^{\prime}$. Analogously, an effect $d \in \mathrm{Eff}(\mathrm{BC})$ is faithful for $\mathrm{B}$ if given two transformations $\mathcal{A}, \mathcal{A}^{\prime} \in$ Transf(A, B), the condition $\left(\left.d\right|_{\mathrm{BC}} \mathcal{A}=\left(\left.d\right|_{\mathrm{BC}} \mathcal{A}^{\prime}\right.\right.$ implies $\mathcal{A}=\mathcal{A}^{\prime}$.

Remark 1. We observe that in the general framework, without further assumptions, states (preparations) and effects (measurements) are on equal footing, and any proposition proved for states can be proved in the same way for effects. Accordingly, since this paper relies only on the general framework of OPTs, all the results given in terms of states, dilations of states, and sets of dilations of states can be mirrored to results on effects, dilations of effects, and sets of dilations of effects, respectively. In the next Section 2.1 we present some significant classes of OPTs that are obtained enriching the present framework with one or more properties, such as the possibility of performing the tomography of states using only local operations, or the possibility of obtaining an arbitrary mixed state as the marginal of a pure one. Among the properties discussed in the following there is also causality, which induces an asymmetry in the structure of states and effects of the theory. Indeed, as it happens in both classical and quantum theory, causality forces the existence of a unique deterministic effect, while the set of states typically presents several deterministic elements also in the presence of causality.

\subsection{Relevant classes of OPTs}

A frequently highlighted property within the wider scenario of OPTs is that of multipartite states discrimination via local measurements:

Definition 9 (Local discriminability). It is possible to discriminate between any pair of states of composite systems using only local measurements. Mathematically, given two joint states $\Psi, \Psi^{\prime} \in \mathrm{St}(\mathrm{AB})$ with $\Psi \neq \Psi^{\prime}$, there exist two effects $a \in \operatorname{Eff}(\mathrm{A})$ and $b \in \operatorname{Eff}(\mathrm{B})$, such that

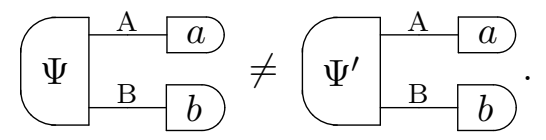

Notice that the names local discriminability and local tomography are used interchangeably in the literature. Also in this manuscript we will consider the two names as synonymous.

Two relevant consequences of local discriminability are: i) the local characterization of transformations, stating that the local behaviour of a transformation is sufficient to fully characterize the transformation itself; ii) the atomicity of parallel composition. Here we report those two features for the convenience of the reader.

Proposition 1 (Local characterization of transformations). If local discriminability holds, then for any two transformations $\mathcal{A}, \mathcal{A}^{\prime} \in$ $\operatorname{Transf}(\mathrm{A}, \mathrm{B})$, the condition $\left.\mathcal{A} \mid \rho)_{\mathrm{A}}=\mathcal{A}^{\prime} \mid \rho\right)_{\mathrm{A}}$ for every $\rho \in \operatorname{St}(\mathrm{A})$ implies $\mathcal{A}=\mathcal{A}^{\prime}$.

See Ref. [10] for the proof.

Proposition 2 (Atomicity of parallel composition). If an OPT satisfies local discriminability then the parallel composition of atomic transformations is atomic.

For the proof of the above proposition see Ref. [22]. We observe that an OPT with local discriminability allows for tomography of multipartite states using only local measurements. In an OPT with local discriminability, the linear space of effects of a composite system is the tensor product of the linear spaces of effects of the component systems, namely $\operatorname{Eff}(\mathrm{AB})_{\mathbb{R}} \equiv \operatorname{Eff}(\mathrm{A})_{\mathbb{R}} \otimes \operatorname{Eff}(\mathrm{B})_{\mathbb{R}}$. Thus, any bipartite effect $c \in \operatorname{Eff}(\mathrm{AB})$ can be written as a linear combination of product effects, and every probability $(c \mid \rho)_{\mathrm{AB}}$, for $\rho \in$ $\mathrm{St}(\mathrm{AB})$, can be computed as a linear combination of the probabilities $\left(\left(\left.a\right|_{\mathrm{A}} \otimes\left(\left.b\right|_{\mathrm{B}}\right) \mid \rho\right)_{\mathrm{AB}}\right.$ arising from a finite set of product effects. The same holds for the linear space of states and in an OPT with local discriminability the parallel composition of two states (effects) can be understood as a tensor product. Finally, the relation $\operatorname{dim}(\mathrm{AB})=\operatorname{dim}(\mathrm{A}) \operatorname{dim}(\mathrm{B})$ between the linear dimension of the set of states/effects holds, whereas for theories without local discriminability it holds $\operatorname{dim}(\mathrm{AB})>\operatorname{dim}(\mathrm{A}) \operatorname{dim}(\mathrm{B})$.

Recently it has been shown that relevant physical theories, such as the Fermionic theory [16], can be described in the OPT framework relaxing the property of local discriminability $[17,18]$. The most general scenario for OPTs that exhibit a finite degree of holism is that of OPTs with nlocal discriminability for some $n \in \mathbb{N}$ [23]: 
Definition 10 ( $n$-local discriminability). A theory satisfies n-local discriminability if whenever two states $\rho$ and $\rho^{\prime}$ are different, there exist a $n$ local effect $b$ such that $(b \mid \rho) \neq\left(b \mid \rho^{\prime}\right)$. We say that an effect is $n$-local if it can be written as a conic combination of tensor products of effects that are at most n-partite.

Two notable examples are indeed Fermionic quantum theory and real quantum computation $[23,17,18]$ that are both 2-local tomographic.

Another relevant class of OPTs is that of theories with purification $[10,24]$. As a result of this paper we will show (Proposition 9) that the set of convex OPTs with purification is strictly smaller than the set of OPTs that satisfy no-information without disturbance. Moreover, we will see that a weak version of purification, which does not require the uniqueness (as in quantum theory) but just the existence of a purification for each state, is enough to imply no-information without disturbance together with the convexity assumption. Accordingly, we define the following class of OPTs.

Definition 11 (States purification). We say that an OPT satisfies states purification if for every system $\mathrm{A}$ and for every state $\rho \in \mathrm{St}(\mathrm{A})$, there exists a system $\mathrm{B}$ and a pure state $\Psi \in \mathrm{St}(\mathrm{AB})$ which is a dilation of $\rho$.

We will prove that also the analogous notion of purification for effects, provided in the following, is sufficient to guarantee no-information without disturbance.

Definition 12 (Effects purification). We say that an OPT satisfies effects purification if for every system A and for every effect $a \in \operatorname{Eff}(\mathrm{A})$, there exists a system $\mathrm{B}$ and an atomic effect $c \in \operatorname{Eff}(\mathrm{AB})$ that is a dilation of $a$.

As already noticed, the above definitions do not require the purification to be unique up to reversible transformations on the purifying system.

The last relevant class of OPTs that we point out is that of causal theories:

Definition 13 (Causal OPTs). The probability of preparation events in a closed circuit is independent of the choice of observations.

Mathematically, if $\left\{\rho_{i}\right\}_{i \in \mathrm{X}} \subset \mathrm{St}(\mathrm{A})$ is a preparation test, then the conditional probability of the preparation $\rho_{i}$ given the choice of the observation test $\left\{a_{j}\right\}_{j \in \mathrm{Y}}$ is the marginal

$$
\operatorname{Pr}\left(i \mid\left\{a_{j}\right\}\right):=\sum_{j \in \mathrm{Y}}\left(a_{j} \mid \rho_{i}\right)_{\mathrm{A}} .
$$

In a causal theory the marginal probability $\operatorname{Pr}\left(i \mid\left\{a_{j}\right\}\right)$ is independent of the choice of the observation test $\left\{a_{j}\right\}:$ if $\left\{a_{j}\right\}_{j \in \mathrm{Y}}$ and $\left\{b_{k}\right\}_{k \in Z}$ are two different observation tests, then one has $\operatorname{Pr}\left(i \mid\left\{a_{j}\right\}\right)=\operatorname{Pr}\left(i \mid\left\{b_{k}\right\}\right)$.

The present notion of causality is simply the Einstein causality expressed in the language of OPTs. As proved in Ref. [10] causality is equivalent to the existence a unique deterministic effect $e_{\mathrm{A}}$. We call the effect $e_{\mathrm{A}}$ the deterministic effect for system A. By definiton in non-causal theories the deterministic effect cannot be unique.

\section{Information and disturbance}

Within the general scenario of operational probabilistic theories, and without further assumptions on the structure of the theory, we aim at defining the notions of non-disturbing and no-information test. These notions have already been investigated for causal theories (Definition 13) that satisfy local discriminability (Definition 9) or states purification (Definition 11). We start highlighting the weakness of previous approaches in cases where the above hypotheses do not hold. The disturbance and the information produced by a test on a physical system A are commonly defined in relation to measurements and states of the system A only, disregarding the action of the same test on an enlarged systems $\mathrm{AB}$.

A test $\left\{\mathcal{A}_{i}\right\}_{i \in \mathrm{X}}$ on system $\mathrm{A}$ is usually said to be non-disturbing if for every $\rho \in \mathrm{St}(\mathrm{A})$ one has that $\left.\left.\sum_{i} \mathcal{A}_{i} \mid \rho\right)_{\mathrm{A}}=\mid \rho\right)_{\mathrm{A}}$. However, this definition is not operationally consistent if applied to theories without local discriminability. A physically relevant example is that of Fermionic theory [16] that, due to the parity superselection rule, is nonlocal tomographic [17, 18] (it is 2-local tomographic according to Definition 10). We can see via a simple example that, for a Fermionic system $\mathrm{A}$, a test $\left\{\mathcal{A}_{i}\right\}_{i \in \mathrm{X}}$ such that $\left.\left.\sum_{i} \mathcal{A}_{i} \mid \rho\right)_{\mathrm{A}}=\mid \rho\right)_{\mathrm{A}}$ for every $\rho \in \mathrm{St}(\mathrm{A})$ still can disturb the states of a composite system AB.

The parity superselection rule on a system $\mathrm{N}_{F}$ of $N$ Fermions forbids any state corresponding to a superposition of vectors belonging to $\mathcal{F}_{N}^{e}$ 
and $\mathcal{F}_{N}^{o}$, representing Fock vector spaces with total even and odd occupation number, respectively. As a consequence, the linearized set of states $\mathrm{St}_{\mathbb{R}}\left(\mathrm{N}_{F}\right)$ splits in the direct sum of two spaces, containing the states with even and odd parity, respectively. It is now convenient to make use of the projectors onto the well-defined parity subspaces $\mathbb{P}_{e}$, for the even space, and $\mathbb{P}_{o}$, for the odd one. Notice that, since $\mathbb{P}_{e} \mathbb{P}_{o}=\mathbb{P}_{o} \mathbb{P}_{e}=0$ any Fermionic state $\rho$ will be of the form $\rho=$ $\mathbb{P}_{e} \rho \mathbb{P}_{e}+\mathbb{P}_{o} \rho \mathbb{P}_{o}$. Consequently the parity test $\left\{\mathbb{P}_{e} \cdot \mathbb{P}_{e}, \mathbb{P}_{o} \cdot \mathbb{P}_{o}\right\}$ leaves every state $\rho \in \operatorname{St}\left(\mathrm{N}_{F}\right)$ unchanged. Intuitively, this seems to suggest that parity can be measured without disturbing. Indeed, this view is in agreement with the notion of disturbance that has been considered in the literature so far.

Consider now a mixed state $\rho \in \operatorname{St}\left(\mathrm{N}_{F}\right)$, with $\rho=p_{e} \rho_{e}+p_{o} \rho_{o}, \rho_{e}$ and $\rho_{o}$ an even and an odd pure state respectively, and $p_{e}+p_{o}=1$. For example, consider the states

$$
\rho_{e}=|00\rangle\left\langle 00\left|, \rho_{o}=\right| 01\right\rangle\langle 01|,
$$

and $p_{e}=p_{o}=1 / 2$, so that

$$
\rho=\frac{1}{2}(|00\rangle\langle 00|+| 01\rangle\langle 01|) .
$$

Since Fermionic theory allows for states purification [17] (see Definition 11), we can always find a state $\Psi \in \mathrm{St}\left(\mathrm{M}_{F}\right)$, with $M>N$ that purifies $\rho$. Since $\Psi$ is pure, it has a definite parity, say even. In our example one can choose

$$
\Psi=\frac{1}{2}(|000\rangle+|011\rangle)(\langle 000|+\langle 011|) .
$$

Therefore, the local test on the system $\mathrm{N}_{F}$ that measures the parity of the system will not disturb the states of $\mathrm{N}_{F}$ but will decohere the state $\Psi$ to a mixed state, then introducing a disturbance. For example, in our case

$$
\begin{aligned}
& \left(\mathbb{P}_{e} \otimes I\right) \Psi\left(\mathbb{P}_{e} \otimes I\right)+\left(\mathbb{P}_{o} \otimes I\right) \Psi\left(\mathbb{P}_{o} \otimes I\right) \\
= & \frac{1}{2}(|000\rangle\langle 000|+| 011\rangle\langle 011|) .
\end{aligned}
$$

In order to avoid the above issue, and to introduce a definition of non-disturbing test that works also for theories without local discriminability, one could say that a test $\left\{\mathcal{A}_{i}\right\}_{i \in \mathrm{X}}$ on system $\mathrm{A}$ is non-disturbing upon input of $\rho \in \mathrm{St}(\mathrm{A})$, if for every $\sigma$ in the refinement set of $\rho$ and every purification $\Psi_{\mathrm{AB}} \in \mathrm{St}(\mathrm{AB})$ of $\sigma$ one has that $\left.\left.\sum_{i} \mathcal{A}_{i} \mid \Psi\right)_{\mathrm{AB}}=\mid \Psi\right)_{\mathrm{AB}}$. This route, which has been proposed in Refs. [10,9], captures the operational meaning of disturbance also for Fermionic systems. However, the definition of Refs. [10, 9] requires purification, and thus cannot be used in theories without purification, e. g. the cases of PR boxes, or the classical theory of information.

Based on the above motivations our proposal is to define the disturbance (and the information) produced by a test in terms of its action on dilations, both of states and effects. This leads to notions of information and disturbance that are completely general and thus do not depend on causality, local discriminability, or purification. This will allow us to prove the no-information without disturbance theorem for a very large class of OPTs. In this Section we first consider the disturbance and the information provided by a test when no restrictions are posed on the states and effects of the theory. The generalization to a scenario where both preparations and measurements are limited to given subsets is presented in Section 4.2.

Definition 14 (Non-disturbing test). Consider a test $\left\{\mathcal{A}_{i}\right\}_{i \in \mathrm{X}}$ on system $\mathrm{A}$. We say that the test is non-disturbing if

$$
\sum_{i} \mathcal{A}_{i}=\mathcal{I}_{\mathrm{A}}
$$

Notice that, following the above definition, the test $\left\{\mathcal{A}_{i}\right\}_{i \in \mathrm{X}}$ is disturbing if there exist $\Psi \in$ $D_{\mathrm{St}(\mathrm{A})}$, and $c \in D_{\mathrm{Eff}(\mathrm{A})}$, such that

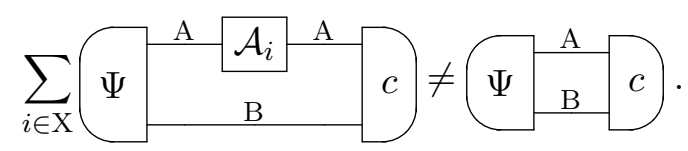

This definition of disturbance thus stresses the effect of a transformation on correlations with remote systems, indeed a test $\left\{\mathcal{A}_{i}\right\}_{i \in \mathrm{X}}$ is nondisturbing if it is operationally equal to the identity transformation of system A, namely it acts as the identity on any possible state and effect of any composite system.

Remark 2. We could have defined a nondisturbing test from $\mathrm{A}$ to $\mathrm{C}$ as follows

$$
\sum_{i} \mathcal{A}_{i}=\mathcal{R}
$$

where $\mathcal{R} \in \operatorname{Transf}(\mathrm{A}, \mathrm{C})$ is a reversible transformation, namely there exists another transformation $\mathcal{W} \in \operatorname{Transf}(\mathrm{C}, \mathrm{A})$ such that $\mathcal{W} \mathcal{R}=\mathcal{I}_{\mathrm{A}}$, and 
$\mathcal{R W}=\mathcal{I}_{\mathrm{C}}$. Indeed if the test provides a systematic reversible transformation on the inputs, then its effect can be trivially corrected by inverting it. The classification of non-disturbing test according to this definition is trivially provided by the classification according to Definition 14. Indeed, the most general non-disturbing test from $\mathrm{A} \rightarrow \mathrm{C}$ is the sequence of tests of the form $\left\{\mathcal{A}_{i} \mathcal{R}\right\}_{i \in \mathrm{X}}$, with $\left\{\mathcal{A}_{i}\right\}_{i \in \mathrm{X}}$ non-disturbing according to Definition 14, and $\mathcal{R} \in \operatorname{Transf}(\mathrm{A}, \mathrm{C})$ reversible.

In the same spirit we can establish if a test provides information. Again a test could provide information both on the input (preparation) and on the output (observation).

Let us consider the task in which Bob wants to extract information on states (effects) of Alice via the test $\mathcal{A}_{\mathrm{X}}:=\left\{\mathcal{A}_{i}\right\}_{i \in \mathrm{X}} \subseteq \operatorname{Transf}(\mathrm{AC})$. Operationally, in the most general case the test $\mathcal{A}_{\mathrm{X}}$ can be used in composite tests made of a preparation test $\Psi_{\mathrm{Y}}:=\left\{\Psi_{j}\right\}_{j \in \mathrm{Y}} \subseteq \operatorname{St}(\mathrm{AB})$, the test $\mathcal{A}_{\mathrm{X}}:=\left\{\mathcal{A}_{i}\right\}_{i \in \mathrm{X}} \subseteq \operatorname{Transf}(\mathrm{AC})$ and an observation test $\boldsymbol{C}_{\mathrm{Z}}:=\left\{c_{k}\right\}_{k \in \mathrm{Z}} \subseteq \operatorname{Eff}(\mathrm{CB})$, leading to the joint probabilities

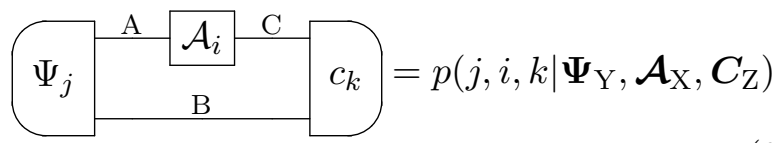

associated with possible outcomes $j, i, k$, and where we explicitly show the dependence of the joint probability distribution $p(j, i, k)$ on the tests composing the circuit.

Within this scenario, Bob can use both the test $\mathcal{A}_{\mathrm{X}}$ and any observation test $\boldsymbol{C}_{\mathrm{Z}}$ in order to extract the information on the inputs, while he can use both the test $\mathcal{A}_{\mathrm{X}}$ and any preparation test $\boldsymbol{\Psi}_{\mathrm{Y}}$ in order to extract the information on the outputs. This leaves room for two inequivalent conditions for a no-information test $\mathcal{A}_{\mathrm{X}}$.

\section{Strong condition for no-information test.}

(a) No-information on inputs: the test $\mathcal{A}_{\mathrm{X}}$ is no-information on inputs if for every preparation test $\boldsymbol{\Psi}_{\mathrm{Y}}$ and for every observation tests $C_{Z}$, the joint probability in Eq. (8) factorizes as

$$
\begin{array}{r}
p\left(j, i, k \mid \boldsymbol{\Psi}_{\mathrm{Y}}, \mathcal{A}_{\mathrm{X}}, \boldsymbol{C}_{\mathrm{Z}}\right) \\
=r\left(i \mid k ; \mathcal{A}_{\mathrm{X}}, \boldsymbol{C}_{\mathrm{Z}}\right) s\left(j, k \mid \boldsymbol{\Psi}_{\mathrm{Y}}, \mathcal{A}_{\mathrm{X}}, \boldsymbol{C}_{\mathrm{Z}}\right),
\end{array}
$$

$$
\begin{array}{llr}
\text { namely } \quad \text { we } & \text { impose } & \text { that } \\
r\left(i \mid j, k ; \boldsymbol{\Psi}_{\mathrm{Y}}, \mathcal{A}_{\mathrm{X}}, \boldsymbol{C}_{\mathrm{Z}}\right) & =r\left(i \mid k ; \mathcal{A}_{\mathrm{X}}, \boldsymbol{C}_{\mathrm{Z}}\right),
\end{array}
$$

where the probability distribution $r$ does not depend on the preparation test $\boldsymbol{\Psi}_{\mathrm{Y}}$ (we remind that it may happen that a probability distribution depends on a given test but not on its outcomes). The interpretation of this condition is that the outcomes of $\mathcal{A}_{\mathrm{X}}$ and their correlations with the outcomes of any observation test do not provide information on the preparation.

(b) No-information on outputs: the test $\mathcal{A}_{\mathrm{X}}$ is no-information on outputs if for every $\boldsymbol{C}_{\mathrm{Z}}$ and for every $\boldsymbol{\Psi}_{\mathrm{Y}}$ the joint probability in Eq. (8) factorizes as

$$
\begin{array}{r}
p\left(j, i, k \mid \boldsymbol{\Psi}_{\mathrm{Y}}, \mathcal{A}_{\mathrm{X}}, \boldsymbol{C}_{\mathrm{Z}}\right) \\
=r\left(i \mid j ; \boldsymbol{\Psi}_{\mathrm{Y}}, \mathcal{A}_{\mathrm{X}}\right) s\left(j, k \mid \boldsymbol{\Psi}_{\mathrm{Y}}, \mathcal{A}_{\mathrm{X}}, \boldsymbol{C}_{\mathrm{Z}}\right),
\end{array}
$$

where the probability distribution $r$ does not depend on the observation test $\boldsymbol{C}_{\mathrm{Z}}$. This condition ensures that the outcomes of $\mathcal{A}_{\mathrm{X}}$ and their correlations with the outcomes of any preparation test do not provide information on the observation.

2. Weak condition for no-information test.

(a) No-information on inputs: the test $\mathcal{A}_{\mathrm{X}}$ is no-information on inputs if for every preparation test $\boldsymbol{\Psi}_{\mathrm{Y}}$ and for every observation test $\boldsymbol{C}_{\mathrm{Z}}$, the joint probability in Eq. (8) is such that

$$
\begin{array}{r}
\sum_{k} p\left(j, i, k \mid \Psi_{\mathrm{Y}}, \mathcal{A}_{\mathrm{X}}, \boldsymbol{C}_{\mathrm{Z}}\right) \\
=r\left(i \mid \mathcal{A}_{\mathrm{X}}, \boldsymbol{C}_{\mathrm{Z}}\right) s\left(j \mid \boldsymbol{\Psi}_{\mathrm{Y}}, \mathcal{A}_{\mathrm{X}}, \boldsymbol{C}_{\mathrm{Z}}\right),
\end{array}
$$

where the probability distribution $r$ does not depend on the preparation test $\boldsymbol{\Psi}_{\mathrm{Y}}$. The interpretation of this condition is that the outcomes of $\mathcal{A}_{\mathrm{X}}$ do not provide information on the preparation, whenever we ignore the outcome of the observation test.

(b) No-information on outputs: the test $\mathcal{A}_{\mathrm{X}}$ is no-information on outputs if for every observation test $\boldsymbol{C}_{\mathrm{Z}}$ and for every preparation test $\boldsymbol{\Psi}_{\mathrm{Y}}$, the joint probability in Eq. (8) is such that

$$
\begin{array}{r}
\sum_{j} p\left(j, i, k \mid \Psi_{\mathrm{Y}}, \mathcal{A}_{\mathrm{X}}, \boldsymbol{C}_{\mathrm{Z}}\right)= \\
r\left(i \mid \boldsymbol{\Psi}_{\mathrm{Y}}, \mathcal{A}_{\mathrm{X}}\right) s\left(k \mid \boldsymbol{\Psi}_{\mathrm{Y}}, \mathcal{A}_{\mathrm{X}}, \boldsymbol{C}_{\mathrm{Z}}\right),
\end{array}
$$


where the probability distribution $r$ does not depend on the observation test $\boldsymbol{C}_{\mathrm{Z}}$. This means that the outcomes of $\mathcal{A}_{\mathrm{X}}$ do not provide information on the observation, whenever we ignore the outcome of the preparation test.

It is elementary to see that $1 \mathrm{a} \Rightarrow 2 \mathrm{a}$ (and $1 \mathrm{~b} \Rightarrow 2 \mathrm{~b}$ ), namely the strong no-information condition implies the weak one, both on inputs and outputs. In the literature on no-information without disturbance in quantum theory the authors take the weak notion 2a of no-information test (only on inputs since the quantum theory is causal). Here, we also choose the conditions 2 as expressed in the next definition (the equivalence between the weak conditions 2 and Definition 15 is proved in Appendix B). The motivation for this choice is that if an OPT satisfies no-information without disturbance according to conditions 2, then it also satisfies no-information without disturbance in the strongest sense of conditions 1 (see Remark 4 in the following).

Definition 15 (No-information test). A test $\left\{\mathcal{A}_{i}\right\}_{i \in \mathrm{X}}$ with events $\mathcal{A}_{i} \in \operatorname{Transf}(\mathrm{A}, \mathrm{C})$ is a noinformation test if, for every choice of deterministic effect $e_{\mathrm{CB}}$ and deterministic state $\omega_{\mathrm{AB}}$, there exists a deterministic effect $f_{\mathrm{AB}}$ and a deterministic state $\nu_{\mathrm{CB}}$ such that for every $i \in \mathrm{X}$ one has

$$
\begin{aligned}
\left(\left.e\right|_{\mathrm{CB}} \mathcal{A}_{i}\right. & =p_{i}(e)\left(\left.f\right|_{\mathrm{AB}},\right. \\
\left.\mathcal{A}_{i} \mid \omega\right)_{\mathrm{AB}} & \left.=q_{i}(\omega) \mid \nu\right)_{\mathrm{CB}} .
\end{aligned}
$$

According to Eq. (11) (that coincides with Eq. (9) in the weak condition of item 2a), the test $\left\{\mathcal{A}_{i}\right\}_{i \in \mathrm{X}}$ does not provide information upon any possible input state. However, the probability distribution $p_{i}(e)$ might in principle provide information about the effect $e$. On the other hand according to Eq. (12) (that coincides with Eq. (10) in the weak condition of item 2b), the test $\left\{\mathcal{A}_{i}\right\}_{i \in \mathrm{X}}$ does not provide information upon output of any possible effect, while the probability distribution $q_{i}(\omega)$ might in principle provide information about the state $\omega$. The conjunction of the two conditions implies that no-information is provided by the test about $D_{\mathrm{St}(\mathrm{A})}$ and $D_{\mathrm{Eff}(\mathrm{A})}$, namely about any possible input state and output effect of any dilated system. The last statement is proved in the following lemma.
Lemma 1. Let the test $\left\{\mathcal{A}_{i}\right\}_{i \in \mathrm{X}}$ with events $\mathcal{A}_{i} \in$ $\operatorname{Transf}(\mathrm{A}, \mathrm{C})$ be a no-information test. Then one has

$$
\begin{aligned}
\left(\left.e\right|_{\mathrm{CB}} \mathcal{A}_{i}\right. & =r_{i}\left(\left.f\right|_{\mathrm{AB}},\right. \\
\left.\mathcal{A}_{i} \mid \omega\right)_{\mathrm{AB}} & \left.=r_{i} \mid \nu\right)_{\mathrm{CB}} .
\end{aligned}
$$

Proof. By Eqs. (11) and (12) one has

$$
\left(\left.e\right|_{\mathrm{CB}} \mathcal{A}_{i} \mid \omega\right)_{\mathrm{AB}}=p_{i}(e)=q_{i}(\omega)=r_{i},
$$

where we used the fact that $e, f$ and $\omega, \nu$ are respectively deterministic effects and deterministic states.

Remark 3. Notice that in Eq. (11) the probability of the transformation $\mathcal{A}_{i} \forall i \in \mathrm{X}$ generally depends on the deterministic effect $e_{\mathrm{CB}}$, this accounting for non-causal theories. In the more general case in which also the deterministic effect $f_{\mathrm{AB}}$ on the right hand side of Eq. (11) depends on $i \in \mathrm{X}$, the test $\left\{\mathcal{A}_{i}\right\}_{i \in \mathrm{X}}$ would provide information on the system state (this would happen, however, only for probabilistic states). An analogous argument holds for $\nu$ in Eq. (12).

\subsection{No-information without disturbance}

In this section we state the condition of noinformation without disturbance and introduce criteria for it to be satisfied by an OPT.

Definition 16 (OPT with no-information without disturbance). We say that an OPT satisfies no-information without disturbance if, for every system $\mathrm{A}$, and every test $\left\{\mathcal{A}_{i}\right\}_{i \in \mathrm{X}} \subseteq \operatorname{Transf}(\mathrm{A})$, if the test is non-disturbing then it is a noinformation test.

Theorem 1. An OPT satisfies no-information without disturbance if and only if the identity transformation is atomic for every system of the theory.

Proof. We start proving that if an OPT satisfies no-information without disturbance then the identity transformation is atomic. Consider a system $\mathrm{A}$ of the theory, and a refinement $\left\{\mathcal{A}_{i}\right\}_{i \in \mathrm{X}}$ $\left(\mathcal{A}_{i} \in \operatorname{Transf}(\mathrm{A})\right.$ for every $\left.i \in \mathrm{X}\right)$ of the identity map $\mathcal{I}_{\mathrm{A}}=\sum_{i} \mathcal{A}_{i}$ for system $\mathrm{A}$. The test $\left\{\mathcal{A}_{i}\right\}_{i \in \mathrm{X}}$ is clearly non-disturbing, therefore by hypothesis it is a no-information test. By definition of noinformation test, and using Lemma 1, we know 
that for every deterministic effect $e_{\mathrm{CB}}$, and deterministic state $\omega_{\mathrm{AB}}$, there exists a deterministic effect $f_{\mathrm{AB}}$ and a deterministic state $\nu_{\mathrm{AB}}$ such that for every $i \in \mathrm{X}$ one has $\left(\left.e\right|_{\mathrm{AB}} \mathcal{A}_{i}=r_{i}\left(\left.f\right|_{\mathrm{AB}}\right.\right.$, and $\left.\left.\mathcal{A}_{i} \mid \omega\right)_{\mathrm{AB}}=r_{i} \mid \nu\right)_{\mathrm{AB}}$. Summing both sides of the last equation over the index $i \in \mathrm{X}$, and remembering that $\sum_{i \in \mathrm{X}} r_{i}=1$, we find that $e=f$ and $\omega=\nu$. Therefore, the no-information condition is

$$
\begin{gathered}
\left(\left.e\right|_{\mathrm{AB}} \mathcal{A}_{i}=r_{i}\left(\left.e\right|_{\mathrm{AB}},\right.\right. \\
\left.\left.\mathcal{A}_{i} \mid \omega\right)_{\mathrm{AB}}=r_{i} \mid \omega\right)_{\mathrm{AB}},
\end{gathered}
$$

for every deterministic effect $e_{\mathrm{AB}}$ and for every deterministic state $\omega_{\mathrm{AB}}$. Consider now an arbitrary pure state $\Psi \in \mathrm{St}(\mathrm{AB})$ (the same proof can be done choosing an arbitrary atomic effect $c \in \operatorname{Eff}(\mathrm{AB}))$. Since $\left.\left.\sum_{i} \mathcal{A}_{i} \mid \Psi\right)_{\mathrm{AB}}=\mid \Psi\right)_{\mathrm{AB}}$, it follows that

$$
\left.\left.\mathcal{A}_{i} \mid \Psi\right)_{\mathrm{AB}}=\lambda_{i}(\Psi) \mid \Psi\right)_{\mathrm{AB}}, \quad \sum_{i} \lambda_{i}(\Psi)=1,
$$

where the coefficients $\lambda_{i}(\Psi)$ generally depend on the state $\Psi$. However, for each pure state $\Psi$ there exists a deterministic effect $e^{\Psi} \in \operatorname{Eff}(\mathrm{AB})$ such that $\left(e^{\Psi} \mid \Psi\right) \neq 0$. Upon applying the deterministic effect $e^{\Psi}$ on both sides of Eq. (17), we get

$$
\left(e^{\Psi}\left|\mathcal{A}_{i}\right| \Psi\right)_{\mathrm{AB}}=\lambda_{i}(\Psi)\left(e^{\Psi} \mid \Psi\right)_{\mathrm{AB}}
$$

Now, applying both sides of Eq. (15) to $\Psi$, we get

$$
\left(e^{\Psi}\left|\mathcal{A}_{i}\right| \Psi\right)_{\mathrm{AB}}=r_{i}\left(e^{\Psi} \mid \Psi\right)_{\mathrm{AB}},
$$

and comparing the last two identities, considering that $\left(e^{\Psi} \mid \Psi\right)_{\mathrm{AB}} \neq 0$, we obtain

$$
\lambda_{i}(\Psi)=r_{i}, \quad \forall i \in \mathrm{X} .
$$

Since this holds true for every pure state $\Psi$, we conclude that $\lambda_{i}(\Psi)$ is independent of $\Psi$. Then $\left.\left.\mathcal{A}_{i} \mid \rho\right)_{\mathrm{AB}}=r_{i} \mid \rho\right)_{\mathrm{AB}}, \forall \rho \in \mathrm{St}(\mathrm{AB})$, proving that $\mathcal{A}_{i}=r_{i} \mathcal{I}_{\mathrm{A}}$. Notice that we implicitly assumed that the probabilities $r_{i}$ do not depend on the choice of the system B. Actually this can be proven as shown in Appendix C.

The converse implication, namely that if in an OPT the identity transformation is atomic then a non-disturbing test is no-information, is trivial.

Remark 4. Eq. (8) shows the most general scenario in which a test $\left\{\mathcal{A}_{i}\right\}_{i \in \mathrm{X}}$ can be used to extract information on its inputs or on its outputs. We noticed that two inequivalent definitions of no-information tests are possible, a strong condition 1 , and a weak condition 2 , depending on the features of the joint probability distribution $p(j, i, k)$ of Eq. (8). However, due to the above theorem, if a theory satisfies no-information without disturbance in the weak sense, then a non-disturbing test $\left\{\mathcal{A}_{i}\right\}_{i \in \mathrm{X}} \in$ $\operatorname{Transf}(\mathrm{A})$ is such that $\mathcal{A}_{i}=q_{i} \mathcal{I}_{\mathrm{A}}$, with $\sum_{i} q_{i}=1$. It follows that in Eq. (8) the joint probability distribution $p(j, i, k)$ is of the form $p(j, i, k)=$ $q_{i} p(j, k)$, and the test is also no-information in the strong sense.

Besides the atomicity of the identity, we can provide other two equivalent necessary and sufficient conditions for no-information without disturbance.

Proposition 3. An OPT satisfies noinformation without disturbance if and only if for every system there exists an atomic transformation which is either left- or right-reversible.

Proof. We start proving that a theory with an atomic reversible transformation for each system satisfies no-information without disturbance. Let $\mathcal{R} \in \operatorname{Transf}(\mathrm{A}, \mathrm{C})$ be atomic and left-reversible (the right-reversible case is analogous). Then consider a refinement $\mathcal{I}_{\mathrm{A}}=\sum_{i} \mathcal{A}_{i}$, with $\mathcal{A}_{i} \in$ Transf(A) for $i \in \mathrm{X}$, of the identity transformation. By definition of identity map we have that $\mathcal{R} \mathcal{I}_{\mathrm{A}}=\sum_{i} \mathcal{R A}_{i}=\mathcal{R}$, and due to the atomicity of $\mathcal{R}$ it must be $\mathcal{R} \mathcal{A}_{i} \propto \mathcal{R}$ for every $i \in \mathrm{X}$. Since $\mathcal{R}$ is left-reversible (namely there exists $\mathcal{W} \in \operatorname{Transf}(\mathrm{C}, \mathrm{A})$ such that $\left.\mathcal{W} \mathcal{R}=\mathcal{I}_{\mathrm{A}}\right)$ it follows that $\mathcal{A}_{i} \propto \mathcal{I}_{\mathrm{A}}$ for every $i \in \mathrm{X}$, which proves the atomicity of $\mathcal{I}_{\mathrm{A}}$.

The other implication, that in a theory that satisfies no-information without disturbance for every system there exists an atomic transformation which is either left- or right-reversible, is trivial. Indeed, in a theory that satisfies no-information without disturbance the identity, which is both right- and left-reversible, is atomic as proved in Theorem 1.

Proposition 4. An OPT satisfies noinformation without disturbance if and only if for every system every reversible transformation is atomic. 
Proof. We prove that if the theory satisfies noinformation without disturbance, then every reversible transformation is atomic. Indeed, let $\mathcal{R} \in \operatorname{Transf}(\mathrm{A})$ be reversible, and suppose that $\mathcal{R}=\sum_{i \in \mathrm{X}} \mathcal{R}_{i}$ for test $\left\{\mathcal{R}_{i}\right\}_{i \in \mathrm{X}}$. Then, one has

$$
\sum_{i \in \mathrm{X}} \mathcal{R}_{i} \mathcal{R}^{-1}=\mathcal{I}_{\mathrm{A}}
$$

and by Theorem 1 one has that $\mathcal{R}_{i} \mathcal{R}^{-1}=p_{i} \mathcal{I}_{\mathrm{A}}$. Finally, multiplying by $\mathcal{R}$ to the right, we conclude that $\mathcal{R}_{i}=p_{i} \mathcal{R}$, namely the refinements of $\mathcal{R}$ must be trivial. For the converse, it is sufficient to observe that the identity is reversible.

\subsection{Information without disturbance}

In this section we provide the general structure of the state spaces and effect spaces of any theory where some information can be extracted from a system without introducing disturbance. Such information is "classical" in the sense that the measurement is the reading of information that is repeatable and shareable. In particular, for the classical OPT the whole information encoded on a system can be read in this way. The proof of the above statements are based on the following theorem.

Theorem 2. The non redundant atomic refinement of the identity is unique for every system. Moreover, given the non redundant atomic refinement $\left\{\mathcal{A}_{i}\right\}_{i \in \mathrm{X}} \subseteq \operatorname{Transf}(\mathrm{A})$ of the identity $\mathcal{I}_{\mathrm{A}}=\sum_{i} \mathcal{A}_{i}$, one has $\mathcal{A}_{i} \mathcal{A}_{j}=\mathcal{A}_{i} \delta_{i j}$.

Proof. Suppose that the identity transformation of system A allows for two atomic refinements $\mathcal{I}_{\mathrm{A}}=\sum_{i \in \mathrm{X}} \mathcal{A}_{i}$, and $\mathcal{I}_{\mathrm{A}}=\sum_{j \in \mathrm{Y}} \mathcal{B}_{j}$. Since $\sum_{i} \mathcal{A}_{i} \mathcal{B}_{j}=\mathcal{B}_{j}$, from the atomicity of the transformations $\mathcal{B}_{j}$ we get $\mathcal{A}_{i} \mathcal{B}_{j}=c_{i j} \mathcal{B}_{j}$, for some $c_{i j} \geq 0$ such that $\sum_{i \in \mathrm{X}} c_{i j}=1 \forall j \in \mathrm{Y}$. Similarly we get $\mathcal{A}_{i} \mathcal{B}_{j}=d_{i j} \mathcal{A}_{i}$ for some $d_{i j} \geq 0$ such that $\sum_{j \in \mathrm{Y}} d_{i j}=1 \forall i \in \mathrm{X}$. Then $c_{i j} \mathcal{B}_{j}=d_{i j} \mathcal{A}_{i}$. By non redundancy one has that for fixed $j$ there is only one value of $i=i(j)$ such that $c_{i j}>0$, and normalisation gives $c_{i(j) j}=1$. By a similar argument for a fixed $i$ there is $j(i)$ such that $d_{i j(i)}=1$. Then one has $\mathcal{B}_{j}=\mathcal{A}_{i(j)}$. This proves the uniqueness of the non redundant atomic refinement of the identity.

By the same argument as before, for the non redundant atomic refinement of the identity one has $\mathcal{A}_{i} \mathcal{A}_{j}=c_{i j} \mathcal{A}_{j}=d_{i j} \mathcal{A}_{i}$, for some $c_{i j}, d_{i j} \geq 0$ such that $\sum_{i \in \mathrm{X}} c_{i j}=\sum_{j \in \mathrm{X}} d_{i j}=1 \forall i, j \in \mathrm{X}$. By atomicity and non redundancy one must have $c_{i j}=d_{i j}=\delta_{i, j}$.

The above theorem has as a consequence the following structure theorem for OPTs.

Corollary 1. For any pair of systems A, B of an OPT one has the following decomposition of the set of states and of the set of effects of $\mathrm{AB}$

$$
\begin{aligned}
\mathrm{St}(\mathrm{AB}) & =\bigoplus_{(i, j) \in \mathrm{X} \times \mathrm{Y}} \mathrm{St}_{i j}(\mathrm{AB}), \\
\mathrm{Eff}(\mathrm{AB}) & =\bigoplus_{(i, j) \in \mathrm{X} \times \mathrm{Y}} \mathrm{Eff}_{i j}(\mathrm{AB}),
\end{aligned}
$$

where for non redundant atomic decompositions $\left\{\mathcal{A}_{i}\right\}_{i \in \mathrm{X}},\left\{\mathcal{B}_{j}\right\}_{j \in \mathrm{Y}}$ of the identities $\mathcal{I}_{\mathrm{A}}$ and $\mathcal{I}_{\mathrm{B}}$, one has

$$
\begin{gathered}
\left.\left.\left(\mathcal{A}_{i} \otimes \mathcal{B}_{j}\right) \mid \Psi_{i^{\prime} j^{\prime}}\right)=\delta_{i i^{\prime}} \delta_{j j^{\prime}} \mid \Psi_{i j}\right), \\
\left(c_{i^{\prime} j^{\prime}} \mid\left(\mathcal{A}_{i} \otimes \mathcal{B}_{j}\right)=\delta_{i i^{\prime}} \delta_{j j^{\prime}}\left(c_{i j} \mid,\right.\right.
\end{gathered}
$$

for all $\Psi_{i^{\prime} j^{\prime}} \in \mathrm{St}_{i^{\prime} j^{\prime}}(\mathrm{AB})$ and $c_{i^{\prime} j^{\prime}} \in \mathrm{Eff}_{i^{\prime} j^{\prime}}(\mathrm{AB})$.

Remark 5. Notice that from Eq. (22) it trivially follows that for any system A the block decomposition holds

$$
\mathrm{St}(\mathrm{A})=\bigoplus_{i \in \mathrm{X}} \mathrm{St}_{i}(\mathrm{~A}), \quad \mathrm{Eff}(\mathrm{A})=\bigoplus_{i \in \mathrm{X}} \operatorname{Eff}_{i}(\mathrm{~A})
$$

However, Eq. (22) contains the additional information that the decomposition holds in that specific form also for composite systems. This is not a straightforward consequence of the decomposition of local states and local effects, as witnessed by the Fermionic case. Indeed, the state in Eq. (4) does not have definite parity for the two subsystems corresponding to two Fermions on the left and one on the right, hence the state space cannot be of the form in Eq. (22).

Remark 6 . For a theory without atomicity of parallel composition it is possibile that the refinement $\mathcal{A}_{i} \otimes \mathcal{B}_{j}$ in Eq. (23) of $\mathcal{I}_{\mathrm{AB}}$ is not atomic. In such a case one has $\mathrm{St}(\mathrm{AB})=\bigoplus_{k \in \mathrm{Z}} \mathrm{St}_{k}(\mathrm{AB})$, and $\mathrm{St}_{i j}(\mathrm{AB})=\bigoplus_{k \in \mathrm{Z}_{i j}} \mathrm{St}_{k}(\mathrm{AB})$, for some partition $\mathrm{Z}_{i j}$ of $\mathrm{Z}$.

\subsubsection{Full information without disturbance}

In the following we formalise the fact that a theory where any information can be extracted via 
a non-disturbing test must have only classical systems. Let us first define the notion of fullinformation without disturbance.

Definition 17 (Full-information without disturbance). An OPT satisfies full-information without disturbance if for every system $\mathrm{A}$ and every test $\left\{\mathcal{B}_{j}\right\}_{j \in \mathrm{Y}} \subseteq \operatorname{Transf}(\mathrm{A})$ there exists a non disturbing test $\left\{\mathcal{A}_{i}\right\}_{i \in \mathrm{X}} \subseteq \operatorname{Transf}(\mathrm{A})$ (namely $\left.\sum_{i} \mathcal{A}_{i}=\mathcal{I}_{\mathrm{A}}\right)$ such that

$$
\mathcal{B}_{j}=\sum_{i} p(j \mid i) \mathcal{V}_{i j} \mathcal{A}_{i} \mathcal{R}_{i j},
$$

for some probability distribution $p$ and reversible transformations $\mathcal{V}_{i j}, \mathcal{R}_{i j} \in \operatorname{Transf}(\mathrm{A})$.

As a consequence of the above definition we have the following lemma on the structure of atomic maps in a theory with full-information without disturbance.

Lemma 2. Consider an OPT with fullinformation without disturbance. Any atomic transformation $\mathcal{B} \in \operatorname{Transf}(\mathrm{A})$ is of the form

$$
\mathcal{B}=\lambda \mathcal{U} \mathcal{A}_{k}=\lambda \mathcal{A}_{l} \mathcal{U}
$$

where $\mathcal{A}_{k}, \mathcal{A}_{l}$ are atomic transformations in the unique non-redundant refinement of the identity $\mathcal{I}_{\mathrm{A}}=\sum_{i} \mathcal{A}_{i}$ of Theorem $2, \mathcal{U} \in \operatorname{Transf}(\mathrm{A})$ is a reversible transformation and $\lambda \geq 0$.

Proof. Consider a test $\left\{\mathcal{B}_{j}\right\}_{j \in \mathrm{Y}} \subseteq \operatorname{Transf}(\mathrm{A})$ such that $\mathcal{B}=\mathcal{B}_{j}$ for some $j \in \mathrm{Y}$. Due to fullinformation without disturbance (see Eq. (25) in Definition 17 where we now take as non disturbing test $\left\{\mathcal{A}_{i}\right\}_{i \in \mathrm{X}}$ the unique non-redundant atomic refinement of the identity $\mathcal{I}_{\mathrm{A}}=\sum_{i} \mathcal{A}_{i}$ ) and to the atomicity of $\mathcal{B}$ one has $\mathcal{B}=\lambda \mathcal{V} \mathcal{A}_{i} \mathcal{R}$, for some $i \in \mathrm{X}, \lambda \geq 0$ and $\mathcal{V}, \mathcal{R} \in \operatorname{Transf}(\mathrm{A})$ reversible transformations. Consider now the three tests $\left\{\mathcal{V} \mathcal{A}_{i} \mathcal{R}\right\}_{i \in \mathrm{X}},\left\{\mathcal{A}_{i} \mathcal{V} \mathcal{R}\right\}_{i \in \mathrm{X}}$ and $\left\{\mathcal{V} \mathcal{R} \mathcal{A}_{i}\right\}_{i \in \mathrm{X}}$, and observe that $\sum_{i} \mathcal{V} \mathcal{A}_{i} \mathcal{R}=\sum_{i} \mathcal{V} \mathcal{R} \mathcal{A}_{i}=$ $\sum_{i} \mathcal{A}_{i} \mathcal{V} \mathcal{R}=\mathcal{V} \mathcal{R}=\mathcal{U}$, with $\mathcal{U} \in \operatorname{Transf}(\mathrm{A})$ reversible. We then conclude the proof noticing that by Theorem 2 the non redundant atomic refinement of a reversible transformation is unique.

We can now state the main Theorem of this section.

Theorem 3. If an OPT is full-information without disturbance then every system of the theory is classical.
Proof. Consider an arbitrary system A of the theory. Since by hypothesis the identity is not atomic, let $\left\{\mathcal{A}_{i}\right\}_{i \in \mathrm{X}}$ be the unique non-redundant atomic refinement of the identity $\mathcal{I}_{\mathrm{A}}=\sum_{i} \mathcal{A}_{i}$ of Theorem 2. Due to Corollary 1 (and the immediately following remark) the sets of states and effects decompose as in Eq. (24). We now prove that all the blocks in such decompositions must be one-dimensional. To this end we show that any pair of states $\rho, \rho^{\prime} \in \mathrm{St}_{i}(\mathrm{~A})$ is such that $\rho^{\prime} \propto \rho$.

First we show that if an OPT satisfies fullinformation without disturbance then for every non null atomic $\rho \in \mathrm{St}_{i}(\mathrm{~A})$ and atomic $a \in$ $\operatorname{Eff}_{i}(\mathrm{~A})$, one has $(a \mid \rho) \neq 0$. Given such $\rho \in \operatorname{St}_{i}(\mathrm{~A})$ and $a \in \operatorname{Eff}_{i}(\mathrm{~A})$ consider the transformation $\mid \rho)(a \mid \in \operatorname{Transf}(\mathrm{A})$, that is generally not atomic. Due to Lemma 2 all the atomic refinements of the above transformation are of the form

$$
\mid \rho)\left(a \mid=\sum_{j} \lambda_{j} \mathcal{U}_{j} \mathcal{A}_{i}\right.
$$

where each $\mathcal{A}_{i}$ is the element of the non redundant refinement of the identity $\mathcal{I}_{\mathrm{A}}$ such that $\left.\left.\mathcal{A}_{i} \mid \rho\right)=\mid \rho\right)$, and $\left(a \mid \mathcal{A}_{i}=\left(a \mid, \lambda_{j}>0\right.\right.$, and the $\mathcal{U}_{j}$ are reversible trasformations. Applying both sides of Eq. (26) to the state $\rho \in \mathrm{St}_{i}(\mathrm{~A})$ one has $\left.\mid \rho)(a \mid \rho)=\sum_{j} \lambda_{j} \mathcal{U}_{j} \mid \rho\right)$. Reminding that the $\mathcal{U}_{j}$ are all reversible, $\lambda_{j}>0$ and $\rho$ is non-null, one concludes that the right hand side cannot be null, and this proves that also the pairing $(a \mid \rho)$ is nonnull.

Let us apply the transformation in Eq. (26) to another arbitrary atomic state $\rho^{\prime} \in \mathrm{St}_{i}(\mathrm{~A})$. Since $\left.\left.\mathcal{A}_{i} \mid \rho^{\prime}\right)=\mid \rho^{\prime}\right)$ one finds $\left.\left.\mid \rho\right)\left(a \mid \rho^{\prime}\right)=\sum_{j} \lambda_{j} \mathcal{U}_{j} \mid \rho^{\prime}\right)$, for some $\lambda_{j}>0$ and $\mathcal{U}_{j}$ reversible transformations. As shown above it is $\left(a \mid \rho^{\prime}\right) \neq 0$, and using the atomicity of $\rho$ one has $\left.\left.\mid \rho^{\prime}\right) \propto \mathcal{U}_{j}^{-1} \mid \rho\right)$ for every $j$. Since this holds true for every atomic state $\rho^{\prime} \in \mathrm{St}_{i}(\mathrm{~A})$, one has proved that all atomic states (and then all states) in $\mathrm{St}_{i}(\mathrm{~A})$ are proportional to the same atomic state, let's say $\left.\mathcal{U}_{j_{0}}^{-1} \mid \rho\right)$ for some $j_{0}$. Via an analogous argument one can see that all effects in $\operatorname{Eff}_{i}(\mathrm{~A})$ are proportional to the same atomic effect.

Remark 7. We remind that a system is classical when all its pure states are jointly perfectly discriminable. In this case the base of the conic hull of the pure states of each system is a simplex, which corresponds to a subset of the set of 
states for a convex theory. A special case of theory whose systems are all classical is the usual classical information theory, where indeed one has full-information without disturbance. On the other hand, even when all systems are classical, the theory can differ from classical information theory e. g. in the rule for systems composition. For example there exist OPTs whose systems are all clssical but that do not satisfy local discriminability (see Ref. [25]).

\section{Information and disturbance with re- stricted input and output}

In this section we extend our previous results to study the relation between disturbance and information when both input states and output effects are limited to some given subsets. To this end we first introduce the basics notion of identical transformations upon restricted input and output resources.

\subsection{Operational identities between transforma- tions}

As expressed in Eq. (1), two transformations $\mathcal{A}, \mathcal{A}^{\prime} \in \operatorname{Transf}(\mathrm{A}, \mathrm{B})$ of an OPT are said to be operationally equal if for every system $\mathrm{C}$ and for every state $\Psi \in \operatorname{St}(\mathrm{AC})$ one has $\mathcal{A} \mid \Psi)_{\mathrm{AC}}=$ $\left.\mathcal{A}^{\prime} \mid \Psi\right)_{\text {AC }}$. However, two non-identical maps $\mathcal{A}, \mathcal{A}^{\prime} \in \operatorname{Transf}(\mathrm{A}, \mathrm{B})$ could behave in the same way when their action is restricted to a relevant subclass of states.

The notion of identical transformation upon input of a state $\rho \in \mathrm{St}(\mathrm{A})$ has been already introduced in the literature (see Refs. [26, 9] and references therein):

Definition 18 (Equal transformations upon input of $\rho)$. We say that two transformations $\mathcal{A}, \mathcal{A}^{\prime} \in \operatorname{Transf}(\mathrm{A}, \mathrm{B})$ are equal upon input of $\rho \in \operatorname{St}(\mathrm{A})$, and write $\mathcal{A}=\rho \mathcal{A}^{\prime}$, if for every $\sigma \in \operatorname{Ref}_{\rho}$ we have that $\mathcal{A} \sigma=\mathcal{A}^{\prime} \sigma$.

Remark 8 (Operational interpretation of equality upon input). The equality upon input of a state $\rho$ was originally introduced for quantum theory in Ref. [26], where the authors extended the equality to the whole support of the chosen density matrix $\rho$. Within the OPT framework the equality upon input of $\rho$ is instead extended to the refinement set $\operatorname{Ref}_{\rho}$ [9]. This choice can be easily motivated in operational terms: the equality $\mathcal{A}={ }_{\rho} \mathcal{A}^{\prime}$ means that the two maps $\mathcal{A}$ and $\mathcal{A}^{\prime}$ are indistinguishable on the state $\rho$, independently of how it has been prepared. Suppose that the state $\rho$ is prepared by Alice as $\rho=\sum_{i \in \mathrm{X}} \sigma_{i}$, for some refinement of $\rho$. Even Alice, using her knowledge of the preparation cannot distinguish between $\mathcal{A}$ and $\mathcal{A}^{\prime}$.

From Proposition 1 we know that the local action of a map is sufficient to determine the map itself if the OPT satisfies local discriminability (see Definition 9). However, for theories without local discriminability the local action of a transformation might not be sufficient to characterize it. According to Definition 18, then, even if $\mathcal{A}={ }_{\rho} \mathcal{A}^{\prime}$, still the maps $\mathcal{A}$ and $\mathcal{A}^{\prime}$ could act differently upon input of dilations of $\rho$, namely it could be $\left.\mathcal{A} \mid \Psi)_{\mathrm{AC}} \neq \mathcal{A}^{\prime} \mid \Psi\right)_{\mathrm{AC}}$, for some $\Psi \in \operatorname{Ref}_{D_{\rho}}$. In this case the difference between $\mathcal{A}$ and $\mathcal{A}^{\prime}$ would go undetected if their action on system A only is considered. For this reason we introduce the notion of equal transformations upon input of dilations of a state $\rho$.

Definition 19 (Equal transformations upon input of $\left.D_{\rho}\right)$. Given a state $\rho \in \mathrm{St}(\mathrm{A})$, we say that two transformations $\mathcal{A}, \mathcal{A}^{\prime} \in \operatorname{Transf}(\mathrm{A}, \mathrm{B})$ are equal upon input of $D_{\rho}$, and write $\mathcal{A}={ }_{D_{\rho}} \mathcal{A}^{\prime}$, if $\left.\mathcal{A} \mid \Psi)_{\mathrm{AC}}=\mathcal{A}^{\prime} \mid \Psi\right)_{\mathrm{AC}}$ for every $\Psi \in \operatorname{Ref}_{D_{\rho}}$.

Notice that the above definition requires that two transformations act in the same way on the set $\operatorname{Ref}_{D_{\rho}}$. Due to the absence of no-restriction of preparation tests, it is not true in general that $D_{\operatorname{Ref}_{\rho}}=\operatorname{Ref}_{D_{\rho}}$, as one might expect. The only inclusion that can be proved without further assumptions is $\operatorname{Ref}_{D_{\rho}} \subseteq D_{\operatorname{Ref}_{\rho}}$, (see Lemma 5 in Appendix D).

Here we show that the two Definitions 18 and 19 coincide for causal OPTs with local discriminability. For this purpose we first need the following lemma.

Lemma 3. In a causal OPT, if $\Psi \in \mathrm{St}(\mathrm{AB})$, with $\Psi \in \operatorname{Ref}_{D_{\rho}}$ for some $\rho \in \operatorname{St}(\mathrm{A})$, then

$$
\left\{\left(\left.b\right|_{\mathrm{B}} \mid \Psi\right)_{\mathrm{AB}} \mid b \in \operatorname{Eff}(\mathrm{B})\right\} \subseteq \operatorname{Ref}_{\rho} .
$$

Proof. Since $\Psi \in \operatorname{Ref}_{D_{\rho}}$ there exists a preparation test $\{\Psi, \bar{\Psi}, \Lambda\} \subseteq \mathrm{St}(\mathrm{AB})$ such that $\Psi+\bar{\Psi} \in$ $D_{\rho}$. For an arbitrary $b \in \operatorname{Eff}(\mathrm{B})$, thanks to causality that ensures the existence of a unique 
deterministic effect (see Definition 13), one can construct the test

$$
\begin{aligned}
& \left\{\left(\left.b\right|_{\mathrm{B}} \mid \Psi\right)_{\mathrm{AB}},\left(e-\left.b\right|_{\mathrm{B}} \mid \Psi\right)_{\mathrm{AB}},\right. \\
& \left(\left.b\right|_{\mathrm{B}} \mid \bar{\Psi}\right)_{\mathrm{AB}},\left(e-\left.b\right|_{\mathrm{B}} \mid \bar{\Psi}\right)_{\mathrm{AB}}, \\
& \left.\left(\left.b\right|_{\mathrm{B}} \mid \Lambda\right)_{\mathrm{AB}},\left(e-\left.b\right|_{\mathrm{B}} \mid \Lambda\right)_{\mathrm{AB}}\right\},
\end{aligned}
$$

where $e_{\mathrm{B}}$ is the unique deterministic effect. Since the coarse-graining of the first four elements is $\rho$, we conclude that $\left(\left.b\right|_{\mathrm{B}} \mid \Psi\right)_{\mathrm{AB}} \in \operatorname{Ref}_{\rho}$.

Proposition 5. In a causal OPT with local discriminability, given two transformations $\mathcal{A}, \mathcal{A}^{\prime} \in$ Transf(A, B), the two conditions $\mathcal{A}=\rho \mathcal{A}^{\prime}$ and $\mathcal{A}={ }_{D_{\rho}} \mathcal{A}^{\prime}$ are equivalent.

Proof. We first prove that $\mathcal{A}={ }_{\rho} \mathcal{A}^{\prime} \Rightarrow \mathcal{A}={ }_{D_{\rho}}$ $\mathcal{A}^{\prime}$. Consider an arbitrary $\Psi \in \operatorname{Ref}_{D_{\rho}}$, with $\rho \in \mathrm{St}(\mathrm{A})$. Let for example be $\Psi \in \mathrm{St}(\mathrm{AC})$. By hypothesis we have that $\mathcal{A}=\rho \mathcal{A}^{\prime}$, namely $\left.\mathcal{A} \mid \sigma)_{\mathrm{A}}=\mathcal{A}^{\prime} \mid \sigma\right)_{\mathrm{A}}$ for every $\sigma \in \operatorname{Ref}_{\rho}$. Then, due to Lemma $3, \forall b \in \operatorname{Eff}(\mathrm{B}), \forall c \in \operatorname{Eff}(\mathrm{C})$, we have that $\left(\left.b\right|_{\mathrm{B}}\left(\left.c\right|_{\mathrm{C}}\left(\mathcal{A} \otimes \mathcal{I}_{\mathrm{C}}\right) \mid \Psi\right)_{\mathrm{AC}}=\left(\left.b\right|_{\mathrm{B}}\left(\left.c\right|_{\mathrm{C}}\left(\mathcal{A}^{\prime} \otimes\right.\right.\right.\right.$ $\left.\left.\mathcal{I}_{\mathrm{C}}\right) \mid \Psi\right)_{\mathrm{AC}}$, and by local discriminability (see Definition 9) we conclude that $\left.\left(\mathcal{A} \otimes \mathcal{I}_{\mathrm{C}}\right) \mid \Psi\right)_{\mathrm{AC}}=$ $\left.\left(\mathcal{A}^{\prime} \otimes \mathcal{I}_{\mathrm{C}}\right) \mid \Psi\right)_{\mathrm{AC}}$. Since this holds true for every $\Psi \in \operatorname{Ref}_{D_{\rho}}$, we conclude that $\mathcal{A}={ }_{D_{\rho}} \mathcal{A}^{\prime}$. The converse implication $\mathcal{A}={ }_{D_{\rho}} \mathcal{A}^{\prime} \Rightarrow \mathcal{A}={ }_{\rho} \mathcal{A}^{\prime}$ is trivial.

Dealing also with non-causal theories, where the role of states and effects is interchangeable (see Remark 1) it is in order to introduce the counterpart of the above definition with effects replacing states. Accordingly we define equal transformations upon output of dilations of an effect a.

Definition 20 (Equal transformations upon output of $\left.D_{b}\right)$. Given an effect $b \in \operatorname{Eff}(\mathrm{B})$, we say that two transformations $\mathcal{A}, \mathcal{A}^{\prime} \in \operatorname{Transf}(\mathrm{A}, \mathrm{B})$ are equal upon output of $D_{b}$, and write $\mathcal{A}_{D_{b}}=\mathcal{A}^{\prime}$, if $\left(\left.c\right|_{\mathrm{BC}} \mathcal{A}=\left(\left.c\right|_{\mathrm{BC}} \mathcal{A}^{\prime}\right.\right.$ for every $c \in \operatorname{Ref}_{D_{b}}$.

In the most general case one can define equality of two transformations when both states and effects are limited to two given subsets.

Definition 21 (Equal transformations upon $X, Y)$. We say that two transformations $\mathcal{A}, \mathcal{A}^{\prime} \in$ $\operatorname{Transf}(\mathrm{A}, \mathrm{B})$ are equal upon input of $X \subseteq D_{\mathrm{St}(\mathrm{A})}$ and upon output of $Y \subseteq D_{\mathrm{Eff}(\mathrm{B})}$-or simply upon $X, Y$-and write $\mathcal{A}_{Y}={ }_{X} \mathcal{A}^{\prime}$, if

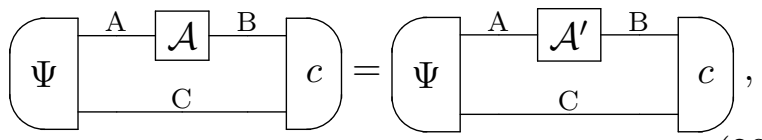

for every $\Psi \in \operatorname{Ref}_{X}$ and for every $c \in \operatorname{Ref}_{Y}$.

As a special case, given a state $\rho \in \mathrm{St}(\mathrm{A})$, and an effect $b \in \operatorname{Eff}(\mathrm{B})$, two transformations $\mathcal{A}, \mathcal{A}^{\prime} \in$ Transf (A, B) are equal upon $D_{\rho}, D_{b}$ when in the last definition we take $X=D_{\rho}$ and $Y=D_{b}$. Accordingly, also Definitions 19 and 20 are special cases of Definition 21, with $\mathcal{A}={ }_{D_{\rho}} \mathcal{A}^{\prime}$ corresponding to the choice $X=D_{\rho}, Y=D_{\mathrm{Eff}(\mathrm{B})}$ and $\mathcal{A}_{D_{b}}=\mathcal{A}^{\prime}$ corresponding to the choice $X=$ $D_{\mathrm{St}(\mathrm{A})}, Y=D_{b}$. Naturally, also the notion of equal transformations $\mathcal{A}=\mathcal{A}^{\prime}$ is the one of Definition 21 with no restrictions on the set states and effects, that is $X=D_{\mathrm{St}(\mathrm{A})}, Y=D_{\mathrm{Eff}(\mathrm{B})}$.

Based on the above identities of transformations, any property of an OPT can be generalized in the upon $X, Y$ scenario. Here we only present the case of two properties that will be used to derive the following results.

In Definition 5 we introduced the notion of atomic events, and we can provide a weaker version of the property of atomicity for transformations.

Definition 22 (Atomic and refinable transformation upon $X, Y$.). A transformation $\mathcal{A} \in$ $\operatorname{Transf}(\mathrm{A}, \mathrm{B})$ is atomic upon input of $X \subseteq D_{\mathrm{St}(\mathrm{A})}$ and upon output of $Y \subseteq D_{\mathrm{Eff}(\mathrm{B})}$-or simply upon $X, Y$-if all its refinements are trivial upon $X, Y$, namely $\mathcal{B} \prec \mathcal{A}$ implies $\mathcal{B}_{Y}={ }_{X} \lambda \mathcal{A}, \lambda \in[0,1]$. Conversely, we say that an event is refinable upon $X, Y$ whenever it is not atomic upon $X, Y$

Again, we mention as a special case the atomicity upon $X=D_{\rho}, Y=D_{b}$ for some state $\rho \in \operatorname{St}(\mathrm{A})$ and effect $b \in \operatorname{Eff}(\mathrm{B})$, which in turn reduces to atomicity upon input of $D_{\rho}$ (atomicity upon output of $D_{b}$ ) when $X=D_{\rho}, Y=D_{\mathrm{Eff}(\mathrm{B})}$ $\left(X=D_{\mathrm{St}(\mathrm{A})}, Y=D_{b}\right)$. Similarly, the usual notion of atomicity corresponds to the choice $X=D_{\mathrm{St}(\mathrm{A})}, Y=D_{\mathrm{Eff}(\mathrm{B})}$.

Finally, the usual notion of faithful state and faithful effect (see Definition 8) can be generalized to the notion of faithful state and faithful effect upon input of $X$ and upon output of $Y$. 
Definition 23 (Faithful state and faithful effect upon $X, Y)$. Consider two arbitrary transformations $\mathcal{A}, \mathcal{A}^{\prime} \in \operatorname{Transf}(\mathrm{A}, \mathrm{B})$ and two subsets $X \subseteq D_{\mathrm{St}(\mathrm{A})}$ and $Y \subseteq D_{\mathrm{Eff}(\mathrm{B})}$. A state $\Psi \in \mathrm{St}(\mathrm{AC})$ is faithful upon input of $X$ and upon output of $Y$-or simply upon $X, Y$-if the condition $\left.\mathcal{A} \mid \Psi)_{\mathrm{AC}}=\mathcal{A}^{\prime} \mid \Psi\right)_{\mathrm{AC}}$ implies $\mathcal{A}_{Y}={ }_{X} \mathcal{A}^{\prime}$. Analogously, an effect $d \in \mathrm{Eff}(\mathrm{BC})$ is faithful upon $X, Y$ if the condition $\left(\left.d\right|_{\mathrm{BC}} \mathcal{A}=\left(\left.d\right|_{\mathrm{BC}} \mathcal{A}^{\prime}\right.\right.$ implies $\mathcal{A}_{Y}={ }_{X} \mathcal{A}^{\prime}$.

The case of faithful state and faithful effect of Definition 8 corresponds to the choice $X=$ $D_{\mathrm{St}(\mathrm{A})}, Y=D_{\mathrm{Eff}(\mathrm{B})}$ in the above definition.

\subsection{Information and disturbance upon $\mathrm{X}, \mathrm{Y}$}

We start generalizing Definition 14 of nondisturbing test:

Definition 24 (Non-disturbing test upon $X, Y)$. Consider a test $\left\{\mathcal{A}_{i}\right\}_{i \in \mathrm{X}}$ on system $\mathrm{A}$. We say that the test is non-disturbing upon input of $X \subseteq$ $D_{\mathrm{St}(\mathrm{A})}$ and upon output of $Y \subseteq D_{\mathrm{Eff}(\mathrm{A})}$-or simply upon $X, Y$ - if

$$
\sum_{i} \mathcal{A}_{i Y}={ }_{X} \mathcal{I}_{\mathrm{A}}
$$

According to Definition 14 a test $\left\{\mathcal{A}_{i}\right\}_{i \in \mathrm{X}}$ is non-disturbing if it is operationally equal to the identity transformation of system A; this is a special case of the above definition when $X=D_{\mathrm{St}(\mathrm{A})}$, and $Y=D_{\text {Eff(A) }}$.

We can now determine if a test $\left\{\mathcal{A}_{i}\right\}_{i \in \mathrm{X}} \subseteq$ Transf(A,C) provides information upon $X \subseteq$ $D_{\mathrm{St}(\mathrm{A})}, Y \subseteq D_{\mathrm{Eff}(\mathrm{C})}$. We first observe that the prescription upon $X, Y$ establishes that (i) we are only interested in getting information on states in $X$ and on effects in $Y$, and (ii) we can only use preparations that involve states in $X$ and measurements that involve effects in $Y$ in order to extract the information. However, a test containing a state in $X$ (or an effect in $Y$ ) may involve events in $\widehat{X}(\widehat{Y})$, where the set $\widehat{Z}$ is the coexistent completion of the set $Z$ as in Definition 6 .

Let us focus on the scheme in Eq. (8), with the state in the set $X$ and effect in the set $Y$. A test that directly provides information on $X$ may give different probability distributions for different elements of $X$, given that one measures an effect $c \in Y$. However, the test could also provide information about $X$ indirectly. This is the case, for example, when for every test $\left\{c_{k}\right\}_{k \in \mathrm{Z}} \subseteq \widehat{Y}$, one has $p(j, i \mid k)=p(i) p(j)$ for $\Psi_{i} \in X$, while factorisation does not occur for $\Psi_{i} \in \widehat{X} \backslash X$. A similar situation can occur when the information is about $Y$, with the roles of preparation and measurement exchanged.

We thus generalize Definition 15 of noinformation test as follows:

Definition 25 (No-information test upon $X, Y$ ). $A$ test $\left\{\mathcal{A}_{i}\right\}_{i \in \mathrm{X}}$ with events $\mathcal{A}_{i} \in \operatorname{Transf}(\mathrm{A}, \mathrm{C})$ is a no-information test upon input of $X \subseteq D_{\mathrm{St}(\mathrm{A})}$ and upon output of $Y \subseteq D_{\mathrm{Eff}(\mathrm{C})}$-or simply upon $X, Y$-if for every choice of deterministic effect $e_{\mathrm{CB}} \in \widehat{Y}$ and deterministic state $\omega_{\mathrm{AB}} \in \widehat{X}$, there exists a deterministic effect $f_{\mathrm{AB}}$ and a deterministic state $\nu_{\mathrm{CB}}$ such that for every $i \in \mathrm{X}$ one has

$$
\begin{gathered}
\left(\left.e\right|_{\mathrm{CB}} \mathcal{A}_{i}=\widehat{X} p_{i}(e)\left(\left.f\right|_{\mathrm{AB}},\right.\right. \\
\left.\left.\mathcal{A}_{i} \mid \omega\right)_{\mathrm{AB} \widehat{Y}}=q_{i}(\omega) \mid \nu\right)_{\mathrm{CB}} .
\end{gathered}
$$

As a special case the above definition coincides with Definition 15, corresponding to $X=D_{\mathrm{St}(\mathrm{A})}$, and $Y=D_{\mathrm{Eff}(\mathrm{A}) \text {. Notice that in this case it is }}$ also $\widehat{X}=X$ and $\widehat{Y}=Y$.

According to Eq. (30), the test $\left\{\mathcal{A}_{i}\right\}_{i \in \mathrm{X}}$ does not provide information upon input of $\widehat{X}$ once the observations are limited to $\widehat{Y}\left(e_{\mathrm{CB}} \in \widehat{Y}\right)$. However, the probability distribution $p_{i}(e)$ might in principle provide information about the effect e. On the other hand according to Eq. (31), the test $\left\{\mathcal{A}_{i}\right\}_{i \in \mathrm{X}}$ does not provide information upon output of $\widehat{Y}$, once the preparations are limited to $\widehat{X}\left(\omega_{\mathrm{AB}} \in \widehat{X}\right)$, while the probability distribution $q_{i}(\omega)$ might in principle provide information about the state $\omega$. The conjunction of the two conditions implies that no-information is provided by the test about $\widehat{X}$ and $\widehat{Y}$ :

Lemma 4. Let the test $\left\{\mathcal{A}_{i}\right\}_{i \in \mathrm{X}}$ with events $\mathcal{A}_{i} \in$ Transf $(\mathrm{A}, \mathrm{C})$ be a no-information test upon $X, Y$. Then one has

$$
\begin{gathered}
\left(\left.e\right|_{\mathrm{CB}} \mathcal{A}_{i}={ }_{\widehat{X}} r_{i}\left(\left.f\right|_{\mathrm{AB}},\right.\right. \\
\left.\left.\mathcal{A}_{i} \mid \omega\right)_{\mathrm{AB}} \widehat{\widehat{Y}}=r_{i} \mid \nu\right)_{\mathrm{CB}} .
\end{gathered}
$$

Proof. By Eqs. (30) and (31), and remembering that $\omega_{\mathrm{AB}} \in \widehat{X}, e_{\mathrm{CB}} \in \widehat{Y}$, one has

$$
\left(\left.e\right|_{\mathrm{CB}} \mathcal{A}_{i} \mid \omega\right)_{\mathrm{AB}}=p_{i}(e)=q_{i}(\omega)=r_{i},
$$

where we used the fact that $e, f$ and $\omega, \nu$ are respectively deterministic effects and deterministic states. 
Here we state the condition of no-information without disturbance upon $X, Y$ :

Definition 26 (No-information without disturbance upon $X, Y)$. Consider a system $\mathrm{A}$ and two subsets $X \subseteq D_{\mathrm{St}(\mathrm{A})}, Y \subseteq D_{\mathrm{Eff}(\mathrm{A})}$. Then the $O P T$ satisfies no-information without disturbance upon input of $X$ and upon output of $Y$-or simply upon $X, Y$-if for every test $\left\{\mathcal{A}_{i}\right\}_{i \in \mathrm{X}} \subseteq \operatorname{Transf}(\mathrm{A})$ that is non-disturbing upon $X, Y$, the test does not provide information upon $X, Y$.

Clearly the above definition generalizes Definition 16 which corresponds to the choice $X=$ $D_{\mathrm{St}(\mathrm{A})}$, and $Y=D_{\mathrm{Eff}(\mathrm{A})}$, namely the theory satisfies no-information without disturbance and any informative test necessary disturbs.

All the results presented in Section 3.1 on noinformation without disturbance can now be extended to the "upon $X, Y$ " scenario. The result of Theorem 1, proving the atomicity of the identity as a necessary and sufficient condition for noinformation without disturbance, is generalized by the following theorem:

Theorem 4. An OPT satisfies no-information without disturbance upon $\widehat{X}, \widehat{Y}$, with $X \subseteq D_{\mathrm{St}(\mathrm{A})}$, $Y \subseteq D_{\mathrm{Eff}(\mathrm{A})}$, if and only if the identity $\mathcal{I}_{\mathrm{A}}$ is atomic upon $\widehat{X}, \widehat{Y}$.

Proof. The proof follows the lines of that of Theorem 1. In the present case Eq. (17) for pure state in $\widehat{X}$ holds upon $\widehat{Y}$. Now, if we apply on both sides a deterministic effect $e_{\mathrm{AB}} \in \hat{Y}$, using Eq. (32) it may happen that $(e \mid \Psi)=0$. The case where this happens for every $e \in \widehat{Y}$ corresponds to a state $\Psi$ which is equal to the null state upon $\widehat{X}, \widehat{Y}$. Considering the remaining cases one concludes that $\left.\left.\mathcal{A}_{i} \mid \rho\right)_{\widehat{Y}}=r_{i} \mid \rho\right), \forall \rho \in \widehat{X}$. Similarly, one concludes that $\left(b \mid \mathcal{A}_{i}={ }_{\widehat{X}} r_{i}(b \mid\right.$, $\forall b \in \widehat{Y}$. These two last conditions imply that $\mathcal{A}_{i}{ }_{\widehat{Y}}={ }_{\widehat{X}} r_{i} \mathcal{I}_{\mathrm{A}}$, corresponding to atomicity of $\mathcal{I}_{\mathrm{A}}$ upon $\widehat{X}, \widehat{Y}$. Also in this case the proof follows straightforwardly from that of Theorem 1 . The opposite implication is trivial.

Analogously, one can generalize the other necessary and sufficient conditions of Section 3.1. One can also provide only sufficient conditions for no-information without disturbance. An example is given in the following proposition:
Proposition 6. An OPT satisfies noinformation without disturbance upon $\widehat{X}, \widehat{Y}$, with $X \subseteq D_{\mathrm{St}(\mathrm{A})}, Y \subseteq D_{\mathrm{Eff}(\mathrm{A})}$, if there exists a pure state $\Psi \in \operatorname{Ref}_{\widehat{X}}$ that is faithful upon $\widehat{X}, \widehat{Y}$. Similarly, an OPT satisfies no-information without disturbance upon $\widehat{X}, \widehat{Y}$ if there exists an atomic effect $b \in \operatorname{Ref}_{\widehat{Y}}$ that is faithful upon $\widehat{X}, \widehat{Y}$.

Proof. We explicitly prove the case of faithful state, since that of faithful effect follows by analogy. Given a system A, let $\Psi \in \operatorname{Ref}_{\widehat{X}}$ be pure and faithful upon $\widehat{X}, \widehat{Y}$ (see Definition 23). Now let the test $\left\{\mathcal{A}_{i}\right\}_{i \in \mathrm{X}} \in \operatorname{Transf}(\mathrm{A})$ be non-disturbing upon $\widehat{X}, \widehat{Y}$, namely $\sum_{i} \mathcal{A}_{i} \widehat{Y}={ }_{\widehat{X}} \mathcal{I}_{\mathrm{A}}$. Then, since $\Psi \in \operatorname{Ref}_{\widehat{X}}$ we have $\left.\left.\sum_{i} \mathcal{A}_{i} \mid \Psi\right)=\mid \Psi\right)$, and since $\Psi$ is pure, there exists a set of probabilities $\left\{p_{i}\right\}_{i \in \mathrm{X}}$ such that $\left.\left.\mathcal{A}_{i} \mid \Psi\right)=p_{i} \mid \Psi\right)$. However, due to the faithfulness of $\Psi$, the map $\mathcal{A} \mapsto \mathcal{A} \mid \Psi)$ is injective upon $\widehat{X}, \widehat{Y}$, and we conclude that $\mathcal{A}_{i}{ }_{\widehat{Y}}={ }_{\widehat{X}} p_{i} \mathcal{I}_{\mathrm{A}}$, and, by definition, the test $\left\{\mathcal{A}_{i}\right\}_{i \in \mathrm{X}} \in \operatorname{Transf}(\mathrm{A})$ does not extract information upon $\widehat{X}, \widehat{Y}$.

As a corollary one has a sufficient condition for no-information without disturbance with no restrictions on inputs and outputs:

Corollary 2. An OPT satisfies no-information without disturbance if for every system A there exists a pure state faithful for $\mathrm{A}$ or an atomic effect faithful for $\mathrm{A}$.

\section{Outlook on no-information without disturbance}

In this last section we analyse the relation between no-information without disturbance and other properties of operational probabilistic theories. We focus on local discriminability (see Definition 9) and purification (see Definitions 11 and 12) that, being typical quantum features, are commonly associated with no-information without disturbance. Here instead we show that noinformation without disturbance can actually be satisfied independently of the above two properties.

No-information without disturbance vs. purification

The following proposition proves that the probabilistic theory [27, 28, 29, 30, 31] corresponding to the PR-boxes model, originally introduced in 
Ref. [32], satisfies no-information without disturbance.

Proposition 7. The PR-boxes theory satisfies no-information without disturbance.

Proof. This can be proved in several ways. For example we show that any system of the theory allows for a reversible atomic transformation and then use Proposition 3. The fact that any system has a reversible atomic transformation follows from the following three points. I) The reversible transformations of the elementary system A of the theory (the convex set of normalized states of A is represented by a square, and the set of reversible transformations of A coincides with the set of symmetries of a square, the dihedral group of order eight $D_{8}$, containing four rotations and four reflections) are atomic [29]. II) From Refs. [33, 34] we know that the set of reversible maps of the $N$-partite system $\mathrm{A}^{\otimes N}$ is generated by local reversible operations plus permutations of the systems. Accordingly, the system $\mathrm{A}^{\otimes N}$ allows for a multipartite reversible transformation $U_{1} \otimes U_{2} \otimes U_{N}$ made of local reversible transformations $U_{i}, i=1, \ldots N$. III) Since PR-boxes satisfy local discriminability, the chosen multipartite transformation is atomic due to the atomicity of parallel composition (see Proposition 2).

As a corollary one has that also PR-boxes with minimal tensor product satisfy no-information without disturbance.

Corollary 3. The PR-boxes theory with minimal tensor product satisfies no-information without disturbance.

Proof. Consider PR-boxes theory with minimal tensor product [14]. We remind that in a probabilistic framework, the minimal tensor product of state sets (or effect sets) of two systems is the operation that yields the set of states (or effects) of the composite system as containing only product states (or product effects) and their probabilistic mixtures. The PR-boxes theory with minimal tensor product has the same elementary system as the PR-boxes theory (with the convex set of normalized states represented by a square), but with composite systems constrained by the minimal tensor product for both states and effects. The proof that this probabilistic theory satisfies no-information without disturbance is as in Proposition 7 (one proves that every system has a reversible atomic transformation and no-information without disturbance follows from Proposition 3).

We can now establish the independence between no-information without disturbance and purification (see also Figure 1).

Proposition 8. No-information without disturbance and states or effects purification are independent.

Proof. We prove that:

1. Purification $\nRightarrow$ No-information without disturbance.

2. No-information without disturbance $\nRightarrow \mathrm{Pu}$ rification

1. Consider deterministic classical theory. This is classical theory where the probabilities of outcomes in any test are either 0 or 1 (see also Ref. [10]). One can easily see that in this probabilistic theory all states are pure and all effects are atomic. As a consequence the theory satisfies both states and effects purification according to Definitions 11 and 12. On the other hand, in this theory all information can be extracted without disturbance.

2. Consider PR-boxes theory with minimal tensor product. On one hand this probabilistic theory satisfies no-information without disturbance (see Corollary 3). On the other hand, one can check that the theory satisfies neither states purification, nor effects purification. First notice that, due to the minimal tensor product prescription, multipartite pure states and atomic effects are tensor products of local pure states and local atomic effects, respectively. Therefore, given a multipartite pure state (atomic effect) all its marginals are also pure states (atomic effects). It follows that a state (effect) that is not pure (atomic) cannot admit of any pure (atomic) dilation. Since the elementary system of PR-boxes with minimal tensor product includes both non pure states and non atomic effects, the theory does not satisfy states or effects purification.

While purification is not enough to imply noinformation without disturbance, in the next 


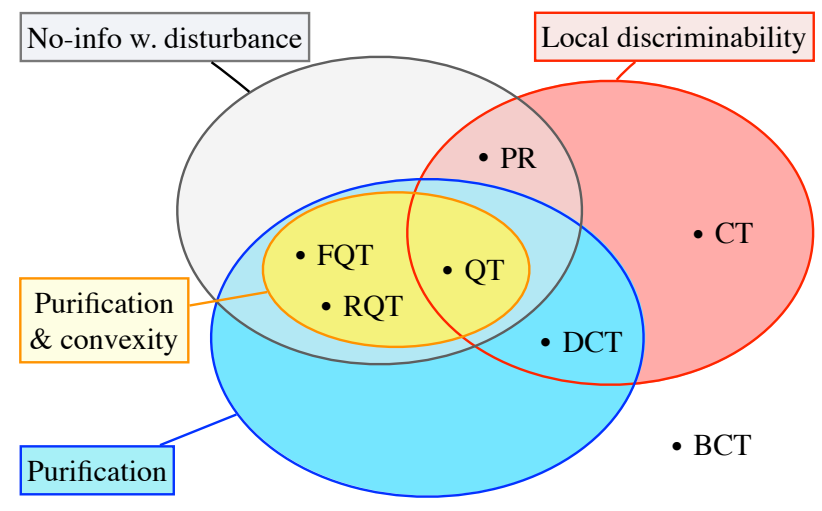

Figure 1: Comparing OPTs that satisfy no-information without disturbance (grey set), local discriminability (red set) and states or effects purification (blue set). Quantum theory (QT) lies at the intersection of the three sets. As proved in Proposition 8 no-information without disturbance and purification are independent features. An example of OPT that satisfies no-information without disturbance but violates purification is the PRboxes theory with minimal tensor product (PR). Moreover, PR-boxes satisfy local discriminability, providing a non-trivial intersection between local discriminability and no-information without disturbance in the absence of purification. On the other hand determinisctic classical theory (DCT) is an example of OPT that satisfies purification but violates no-information without disturbance. As proved in Proposition 9 the set of convex OPTs with purification is a proper subset of OPTs with no-information without disturbance. We observe that no-information without disturbance is also independent of local discriminability, as proved in Proposition 10. Indeed classical theory (CT) satisfies only local discriminability while Fermionic quantum theory (FQT) and real quantum theory (RQT) satisfy only no-information without disturbance. Finally, it has been shown in Ref. [35] that there exist OPTs without local discriminability, that have all systems classical, thus retaining the possibility of extracting all the information without disturbance. An example is the bilocal classical theory (BCT) of the same Ref. [35], which satisfies 2-local discriminability (see Definiton 10).

proposition we show that convex OPTs ${ }^{3}$ that satisfy states or effect purification are a subset of the OPTs with no-information without disturbance (actually they are a proper subset, see also Fig. 1). This provides another useful sufficient condition for no-information without disturbance

\footnotetext{
${ }^{3}$ Actually one might relax the convexity hypothesis with the following weaker condition: If two states belong to a jointly perfectly discriminable set, there exists an element of the set that can be convexly mixed with both. In particular, this is the case if some convex combination of the two states is a state of the theory.
}

(see also Figure 1).

Proposition 9. A convex OPT with states purification or effects purification satisfies noinformation without disturbance.

Proof. We consider the case of states purification (see Definition 11), with the proof for effects purification (see Definition 12) following by analogy according to Remark 1.

Given a convex OPT with states purification suppose that it violates no-information without disturbance, namely there exists a system $\mathrm{A}$ such that $\mathcal{I}_{\mathrm{A}}$ is not atomic. Then let $\mathcal{I}_{\mathrm{A}}=\sum_{i} \mathcal{A}_{i}$, for some atomic non redundant test $\left\{\mathcal{A}_{i}\right\}_{i \in \mathrm{X}} \subseteq$ Transf(A). Let us consider a mixed state $\mathrm{St}(\mathrm{A}) \ni$ $\left.\mid \rho)=\sum_{i \in \mathrm{X}} p_{i} \mid \sigma_{i}\right)$ with $\left.\left.p_{i} \mid \sigma_{i}\right):=\mathcal{A}_{i} \mid \rho\right)$, and $\left\{p_{i}\right\}_{i \in \mathrm{X}}$ a probability distribution with $p_{i}>0$ $\forall i$. Then by Theorem 2 we have $\left.\mathcal{A}_{i} \mid \sigma_{j}\right)=$ $\left.\delta_{i j} \mid \sigma_{i}\right)$. Since the theory allows for purification, let $\Psi \in \mathrm{St}(\mathrm{AB})$ be a purification of $\rho$ for deterministic effect $e \in \operatorname{Eff}(\mathrm{B})$. Now, one one hand since the test $\left\{\mathcal{A}_{i}\right\}_{i \in \mathrm{X}}$ refines the identity, it is $\left.\mid \Psi)_{\mathrm{AB}}=\sum_{i \in \mathrm{X}} \mathcal{A}_{i} \mid \Psi\right)_{\mathrm{AB}}$, and being $\Psi$ pure it must be $\left.\left.\mathcal{A}_{i} \mid \Psi\right)_{\mathrm{AB}}=q_{i} \mid \Psi\right)_{\mathrm{AB}}$, with $\left\{q_{i}\right\}_{i \in \mathrm{X}}$ a probability distribution. On the other hand, for every $i \neq j$ the marginals with deterministic effect $e \in \operatorname{Eff}(\mathrm{B})$ of $\left.\mathcal{A}_{i} \mid \Psi\right)_{\mathrm{AB}}$ and $\left.\mathcal{A}_{j} \mid \Psi\right)_{\mathrm{AB}}$ are perfectly discriminable. But this contradicts the fact that $\left.\mathcal{A}_{i} \mid \Psi\right)_{\mathrm{AB}}$ and $\left.\mathcal{A}_{j} \mid \Psi\right)_{\mathrm{AB}}$ are both proportional to $\Psi$.

No-information without disturbance vs. local discriminability

Turning to the case of local discriminability (see Definition 9), we now show that it is independent of no-information without disturbance (see also Figure 1).

Proposition 10. No-information without disturbance and local discriminability are independent.

Proof. We prove that:

1. Local discriminability $\nRightarrow$ No-information without disturbance,

2. No-information without disturbance $\nRightarrow$ Local discriminability.

1. Classical theory satisfies local discriminability but violates no-information without disturbance, since in this theory all information can be extracted without disturbance. 
2. Fermionic $[16,18,17]$ and real quantum theory $[23,17,18]$ both violate local discriminability as proved in Refs. [23, 17, 18] where it is also shown that they are both 2-local theories according to Definition 10. On the other hand Fermionic and real quantum theories are convex theories with states purification and, due to Proposition 9, both satisfy no-information without disturbance.

Finally, we observe that as a consequence of Corollary 3, and subsequent Remark 7, the classical theory of information is the only theory with local discriminability in which all the information can be extracted without disturbance. However, in the absence of local discriminability, it is still possible to have other theories where all the information can be extracted without disturbance. This has been proved in Ref. [35] where the authors describe an OPT whose systems of any dimension are classical (and then violate noinformation without disturbance), but with a parallel composition that differs from the usual classical one, leading to a violation of local discriminability, more precisely to a 2-local theory according to Definition 10. This theory is interesting because it provides an example of OPT that violates simultaneously no-information without disturbance, local discriminability and purification (see bilocal classical theory (BCT) in Figure 1).

\section{Conclusions}

We have analysed the interplay between information and disturbance for a general operational probabilistic theory, considering the effect of measurements also on correlations with the environment, differently from the traditional approach focused only on the measured system. Indeed, the two resulting notions of disturbance coincide only in special cases, such as quantum theory, as well as every theory that satisfies local discriminability. Our approach is universal for any OPT, including theories without causality, purification or convexity. In this setting we proved that the atomicity of the identity transformation is an equivalent condition for no-information without disturbance.

We have characterized the structure of theories where the identity is not atomic, showing that in this case the information that can be extracted without disturbance is "classical", in the sense that it is sharable and repeatable. On the other hand, we have established that every OPT entails information whose extraction requires disturbance, with the only exception of theories with all systems classical.

While no-information without disturbance is a consequence of convexity along with purification (purification of states or of effects), we proved that purification and no-information without disturbance are independent. Similarly, we have shown that no-information without disturbance and local discriminability are independent properties.

Our results are expected to have immediate applicability to secure key-distribution. Indeed, a physical theory including a system (or even just a set of states of a system) that satisfies no-information without disturbance can guarantee a private and reliable channel for distributing messages. The idea of studying secure keydistribution in a framework more general than the classical and the quantum ones has been proposed in Refs. [12, 14]. In Ref. [12] it has been conjectured that in every theory that is not classical secure key-distribution is possible. The present generalisation of no-information without disturbance to arbitrary OPTs is a first step in proving such a conjecture.

\section{Acknowledgments}

This publication was made possible thanks to the financial support of Elvia and Federico Faggin Foundation.

\section{References}

[1] W. Heisenberg. Über den anschaulichen inhalt der quantentheoretischen kinematik und mechanik. Zeitschrift für Physik, 43(3):172198, Mar 1927. doi:https://doi.org/10. 1007/BF01397280.

[2] Paul Busch, Teiko Heinonen, and Pekka Lahti. Heisenberg's uncertainty principle. Physics Reports, 452(6):155 - 176, 2007. doi:https://doi.org/10.1016/j. physrep.2007.05.006.

[3] Paul Busch, Pekka Lahti, and Reinhard F. Werner. Colloquium: Quantum root-mean- 
square error and measurement uncertainty relations. Rev. Mod. Phys., 86:1261-1281, Dec 2014. doi: https://doi .org/10.1103/ RevModPhys.86.1261.

[4] Christopher A. Fuchs and Asher Peres. Quantum-state disturbance versus information gain: Uncertainty relations for quantum information. Phys. Rev. A, 53:20382045, Apr 1996. doi:https://doi.org/10. 1103/PhysRevA.53.2038.

[5] Giacomo Mauro D'Ariano. On the heisenberg principle, namely on the informationdisturbance trade-off in a quantum measurement. Fortschritte der Physik: Progress of Physics, 51(4-5):318-330, 2003. doi:https: //doi.org/10.1002/prop. 200310045.

[6] Masanao Ozawa. Uncertainty relations for noise and disturbance in generalized quantum measurements. Annals of Physics, 311(2):350 - 416, 2004. doi:https://doi. org/10.1016/j.aop.2003.12.012.

[7] Lorenzo Maccone. Information-disturbance tradeoff in quantum measurements. Phys. Rev. A, 73:042307, Apr 2006. doi:https:// doi.org/10.1103/PhysRevA.73.042307.

[8] Paul Busch. "No Information Without Disturbance": Quantum Limitations of Measurement, pages 229-256. Springer Netherlands, Dordrecht, 2009. doi:https://doi. org/10.1007/978-1-4020-9107-0_13.

[9] Giacomo Mauro D'Ariano, Giulio Chiribella, and Paolo Perinotti. Quantum Theory from First Principles: An Informational Approach. Cambridge University Press, 2017. doi:https://doi.org/10. 1017/9781107338340.

[10] Giulio Chiribella, Giacomo Mauro D'Ariano, and Paolo Perinotti. Probabilistic theories with purification. Phys. Rev. A, 81:062348, Jun 2010. doi:https://doi.org/10.1103/ PhysRevA . 81.062348.

[11] Giulio Chiribella, Giacomo Mauro D'Ariano, and Paolo Perinotti. Quantum from Principles, pages 171-221. Springer Netherlands, Dordrecht, 2016. doi:https://doi.org/ 10.1007/978-94-017-7303-4_6.

[12] Jonathan Barrett. Information processing in generalized probabilistic theories. Physical Review A, 75(3):032304, 2007. doi:https://doi.org/10.1103/ PhysRevA.75.032304.
[13] Gen Kimura, Koji Nuida, and Hideki Imai. Distinguishability measures and entropies for general probabilistic theories. Reports on Mathematical Physics, 66(2):175 - 206, 2010. doi:https://doi.org/10. 1016/S0034-4877(10)00025-X.

[14] Howard Barnum and Alexander Wilce. Information processing in convex operational theories. Electronic Notes in Theoretical Computer Science, 270(1):3 15, 2011. Proceedings of the Joint 5th International Workshop on Quantum Physics and Logic and 4th Workshop on Developments in Computational Models (QPL/DCM 2008). doi:https://doi.org/ $10.1016 / j$. entcs. 2011.01 .002 .

[15] Teiko Heinosaari, Leevi Leppäjärvi, and Martin Plávala. No-free-information principle in general probabilistic theories. Quantum, 3:157, July 2019. doi:https://doi. org/10.22331/q-2019-07-08-157.

[16] Sergey B. Bravyi and Alexei Yu. Kitaev. Fermionic quantum computation. Annals of Physics, 298(1):210 - 226, 2002. doi : https: //doi.org/10.1006/aphy.2002.6254.

[17] Giacomo Mauro D'Ariano, Franco Manessi, Paolo Perinotti, and Alessandro Tosini. The feynman problem and fermionic entanglement: Fermionic theory versus qubit theory. International Journal of Modern Physics A, 29(17):1430025, 2014. doi:https://doi. org/10.1142/S0217751X14300257.

[18] G. M. D'Ariano, F. Manessi, P. Perinotti, and A. Tosini. Fermionic computation is non-local tomographic and violates monogamy of entanglement. EPL (Europhysics Letters), 107(2):20009, jul 2014. doi:https://doi.org/10.1209/ 0295-5075/107/20009.

[19] Corsin Pfister and Stephanie Wehner. An information-theoretic principle implies that any discrete physical theory is classical. Nature Communications, 4:1851 EP -, 05 2013. doi:https://doi.org/10.1038/ ncomms 2821 .

[20] Ryuji Takagi and Bartosz Regula. General resource theories in quantum mechanics and beyond: Operational characterization via discrimination tasks. Phys. Rev. X, 9:031053, Sep 2019. doi:https://doi.org/ 10.1103/PhysRevX.9.031053. 
[21] Bob Coecke, Tobias Fritz, and Robert W. Spekkens. A mathematical theory of resources. Information and Computation, 250:59 - 86, 2016. Quantum Physics and Logic. doi:https://doi.org/10.1016/j. ic. 2016.02.008.

[22] G M D'Ariano, F Manessi, and P Perinotti. Determinism without causality. Physica Scripta, T163:014013, dec 2014. doi:https://doi.org/10.1088/ 0031-8949/2014/t163/014013.

[23] Lucien Hardy and William K Wootters. Limited holism and real-vector-space quantum theory. Foundations of Physics, 42(3):454473, 2012. doi:https://doi.org/10. 1007/s10701-011-9616-6.

[24] G. Chiribella, G. M. D'Ariano, and P. Perinotti. Informational derivation of quantum theory. Phys. Rev. A, 84(012311):012311-012350, 2011. doi:https://doi.org/10.1103/ PhysRevA. 84.012311.

[25] Giacomo Mauro D'Ariano, Marco Erba, and Paolo Perinotti. Classical theories with entanglement. Phys. Rev. A, 101:042118, Apr 2020. doi:https://doi.org/10.1103/ PhysRevA.101.042118.

[26] Michael A. Nielsen and Isaac L. Chuang. Quantum Computation and Quantum Information: 10th Anniversary Edition. Cambridge University Press, 2010. doi:https: //doi.org/10.1017/CB09780511976667.

[27] Jonathan Barrett. Information processing in generalized probabilistic theories. Phys. Rev. A, 75:032304, Mar 2007. doi:https://doi. org/10.1103/PhysRevA.75.032304.

[28] Jonathan Barrett, Noah Linden, Serge Massar, Stefano Pironio, Sandu Popescu, and David Roberts. Nonlocal correlations as an information-theoretic resource. Phys. Rev.
A, 71:022101, Feb 2005. doi:https://doi. org/10.1103/PhysRevA.71.022101.

[29] Giacomo Mauro D'Ariano and Alessandro Tosini. Testing axioms for quantum theory on probabilistic toy-theories. Quantum Information Processing, 9(2):95-141, 2010. doi:https://doi.org/10.1007/ s11128-010-0172-3.

[30] Anthony J Short and Jonathan Barrett. Strong nonlocality: a trade-off between states and measurements. New Journal of Physics, 12(3):033034, mar 2010. doi:https://doi.org/10.1088/ 1367-2630/12/3/033034.

[31] Michele Dall'Arno, Sarah Brandsen, Alessandro Tosini, Francesco Buscemi, and Vlatko Vedral. No-hypersignaling principle. Phys. Rev. Lett., 119:020401, Jul 2017. doi:https://doi.org/10.1103/ PhysRevLett.119.020401.

[32] Sandu Popescu and Daniel Rohrlich. Quantum nonlocality as an axiom. Foundations of Physics, 24(3):379-385, 1994.

[33] David Gross, Markus Müller, Roger Colbeck, and Oscar C. O. Dahlsten. All reversible dynamics in maximally nonlocal theories are trivial. Phys. Rev. Lett., 104:080402, Feb 2010. doi:https://doi.org/10.1103/ PhysRevLett.104.080402.

[34] Sabri W Al-Safi and Anthony J Short. Reversible dynamics in strongly non-local boxworld systems. Journal of Physics A: Mathematical and Theoretical, 47(32):325303, jul 2014. doi:https://doi.org/10.1088/ 1751-8113/47/32/325303.

[35] Giacomo Mauro D'Ariano, Marco Erba, and Paolo Perinotti. Classicality without local discriminability: Decoupling entanglement and complementarity. Phys. Rev. A, 102:052216, Nov 2020. doi:https://doi. org/10.1103/PhysRevA.102.052216. 


\section{A Transformations induced by events}

In the operational framework any event $\mathcal{A}$ induces a map between states. Consider for example the event

$$
\mathrm{A} A \mathrm{~B} .
$$

For every choice of ancillary system $\mathrm{C}$, and for every state $\Psi \in \operatorname{St}(\mathrm{AC})$, the event $\mathcal{A}$ maps the state $\Psi$ to the state given by the following circuit

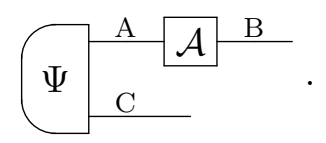

Accordingly, while states and effects are linear functionals over each other, we can always look at an event as a map between states

$$
\left.\mathcal{A}: \mid \Psi)_{\mathrm{AC}} \in \mathrm{St}(\mathrm{AC}) \mapsto \mathcal{A} \mid \Psi\right)_{\mathrm{AC}} \in \mathrm{St}(\mathrm{BC}),
$$

(and similarly as a map between effects, from $\operatorname{Eff}(\mathrm{AC})$ to $\operatorname{Eff}(\mathrm{BC})$ ). The map above can be linearly extended to a map from $\mathrm{St}_{\mathbb{R}}(\mathrm{AC})$ to $\mathrm{St}_{\mathbb{R}}(\mathrm{BC})$ (we denote the extended map with the same symbol $\mathcal{A}$ ) and this extension is unique. Indeed a linear combination of states of $\mathrm{AC}$ is null, say $\left.\sum_{i} c_{i} \mid \Psi_{i}\right)$, if and only if $\sum_{i} c_{i}\left(a \mid \Psi_{i}\right)=0$ for any $a \in \operatorname{Eff}(\mathrm{BC})$. Moreover, since $\left(\left.b\right|_{\mathrm{B}} \mathcal{A} \in \operatorname{Eff}(\mathrm{AC})\right.$ for every $b \in \operatorname{Eff}(\mathrm{B})$, then $\forall b \in \operatorname{Eff}(\mathrm{B})$ we have that $0=\left(\left.b\right|_{\mathrm{B}} \mathcal{A}\left(\sum_{i} c_{i} \mid \Psi_{i}\right)_{\mathrm{AB}}\right)=\sum_{i} c_{i}\left(\left.b\right|_{\mathrm{B}} \mathcal{A} \mid \Psi_{i}\right)_{\mathrm{AB}}=\left(\left.b\right|_{\mathrm{B}} \sum_{i} c_{i} \mathcal{A} \mid \Psi_{i}\right)_{\mathrm{AB}}$, and we finally get $\left.\sum_{i} c_{i} \mathcal{A} \mid \Psi_{i}\right)_{\mathrm{AB}}=0$.

\section{B No-information test}

We show that the weak condition 2 for no-information test is equivalent to one in Definition 15.

We focus now on no-information on the input (the case of no-information on the output following by analogy) and show that Eqs. (9) and (11) are equivalent. We first prove that Eq. (11) implies Eq. (9). To this end we evaluate the left hand side of Eq. (9) using Eq. (11), namely

$$
\begin{array}{r}
\sum_{k} p\left(j, i, k \mid \Psi_{\mathrm{Y}}, \mathcal{A}_{\mathrm{X}}, \boldsymbol{C}_{\mathrm{Z}}\right)=\sum_{k}\left(c_{k}\left|\mathcal{A}_{i}\right| \Psi_{j}\right) \\
=\left(e\left|\mathcal{A}_{i}\right| \Psi_{j}\right)=p_{i}(e)\left(f \mid \Psi_{j}\right) .
\end{array}
$$

Now we notice that $p_{i}(e)$, which is a probability distribution on the outcomes of the test $\mathcal{A}_{\mathrm{X}}$, also depends on the deterministic effect $e=\sum_{k} c_{k}$. Therefore $p_{i}(e)$ is a probability distribution that depends on both the test $\mathcal{A}_{\mathrm{X}}$ and on the observation test $\boldsymbol{C}_{\mathrm{Z}}$ while it does not depend on the preparation test $\boldsymbol{\Psi}_{\mathrm{Y}}$, exactly as the probability distribution $r$ on the right hand side of Eq. (9). Finally, we notice that $\left(f \mid \Psi_{j}\right)$ is a probability distribution on the outcomes of the preparation test $\boldsymbol{\Psi}_{\mathrm{Y}}$, and the deterministic effect $f$ can depend on both tests $\mathcal{A}_{\mathrm{X}}$ and $\boldsymbol{C}_{\mathrm{Z}}$. We then conclude that $\left(f \mid \Psi_{j}\right)$ is a probability distribution that generally depends on all test $\Psi_{\mathrm{Y}}, \mathcal{A}_{\mathrm{X}}$ and $\boldsymbol{C}_{\mathrm{Y}}$, as the probability distribution $s$ on the right hand side of Eq. (9). Now we check the other implication, namely that Eq. (9) implies Eq. (11). Due to Eq. (9), one has

$$
\begin{aligned}
& \left(e\left|\mathcal{A}_{i}\right| \Psi_{j}\right)=\sum_{k}\left(c_{k}\left|\mathcal{A}_{i}\right| \Psi_{j}\right)=\sum_{k} p\left(j, i, k \mid \Psi_{\mathrm{Y}}, \mathcal{A}_{\mathrm{X}}, \boldsymbol{C}_{\mathrm{Z}}\right) \\
& =r\left(i \mid \mathcal{A}_{\mathrm{X}}, \boldsymbol{C}_{\mathrm{Z}}\right) s\left(j \mid \boldsymbol{\Psi}_{\mathrm{Y}}, \mathcal{A}_{\mathrm{X}}, \boldsymbol{C}_{\mathrm{Z}}\right) .
\end{aligned}
$$

First we notice that summing over the index $i$ in the first and last member of Eq. (35) we get $s\left(j \mid \Psi_{\mathrm{Y}}, \mathcal{A}_{\mathrm{X}}, \boldsymbol{C}_{\mathrm{Z}}\right)=\left(f \mid \Psi_{j}\right)$, where $\left(f \mid=\sum_{i}\left(e \mid \mathcal{A}_{i}\right.\right.$ is a deterministic effect depending on tests $\mathcal{A}_{\mathrm{X}}, \boldsymbol{C}_{\mathrm{Z}}$ (indeed $\sum_{i} \mathcal{A}_{i}$ is a deterministic transformation). Therefore one has $\left(e \mid \mathcal{A}_{i}=r\left(i \mid \mathcal{A}_{\mathrm{X}}, \boldsymbol{C}_{\mathrm{Z}}\right)(f \mid\right.$. Since on the left hand side of the last identity the only dependence on the observation test $C_{\mathrm{Z}}$ is through the deterministic effect $e$, one finally gets that $r\left(i \mid \mathcal{A}_{\mathrm{X}}, \boldsymbol{C}_{\mathrm{Z}}\right)$ is of the form $p_{i}(e)$, as in Eq. (11). 


\section{Techical observation}

Given a transformation $\mathcal{A} \in \operatorname{Transf}(\mathrm{A})$ such that for every system $\mathrm{B}$ there exists $p_{\mathrm{B}}$ such that

$$
\left.\mathcal{A} \mid \rho)_{\mathrm{AB}}=p_{\mathrm{B}} \mid \rho\right)_{\mathrm{AB}}
$$

then actually $p_{\mathrm{B}} \equiv p$ cannot depend on the system $\mathrm{B}$. Indeed, choosing $\rho=\tau \otimes \sigma_{\mathrm{B}}$ with arbitrary $\tau \in \mathrm{St}(\mathrm{A})$ and normalised $\sigma \in \mathrm{St}(\mathrm{B})$, and discarding system B on both sides of Eq. (36) $\forall \rho \in \mathrm{St}(\mathrm{AB})$, one obtains $\left.\mathcal{A} \mid \tau)=p_{\mathrm{B}} \mid \tau\right)$. This clearly shows that $p_{\mathrm{B}} \equiv p$.

\section{Techical lemma}

Lemma 5. Given a state $\rho \in \mathrm{St}(\mathrm{A})$ one has $\operatorname{Ref}_{D_{\rho}} \subseteq D_{\operatorname{Ref}_{\rho}}$, where $\operatorname{Ref}_{D_{\rho}}$ denotes the union of the refinements of any state in $D_{\rho}$. Given an effect $a \in \operatorname{Eff}(\mathrm{A})$ one has $\operatorname{Ref}_{D_{a}} \subseteq D_{\operatorname{Ref}_{a}}$, where $\operatorname{Ref}_{D_{a}}$ denotes the union of the refinements of any effect in $D_{a}$.

Proof. Since the proof in the case of states and effects is exactly the same (see Remark 1) we focus on the former. Consider a system $\mathrm{B}$ and a state $\Psi \in \mathrm{St}(\mathrm{AB})$ :

1. $\Psi \in D_{\operatorname{Ref}_{\rho}}$ iff $\left.\left(\left.e\right|_{\mathrm{B}} \mid \Psi\right)_{\mathrm{AB}}=\mid \sigma\right)_{\mathrm{A}}$, with $\sigma \in \operatorname{Ref}_{\rho}$, and $e \in \operatorname{Eff}(\mathrm{B})$ deterministic.

2. $\Psi \in \operatorname{Ref}_{D_{\rho}}$ iff there exists a state $\Omega \in \operatorname{St}(\mathrm{AB})$, with $\Omega \in D_{\rho}$ such that $\Psi \in \operatorname{Ref}_{\Omega}$, i.e. there exists a refinement $\left\{\Psi_{i}\right\}_{i \in \mathrm{X}}$ of $\Omega$ such that $\Psi \in\left\{\Psi_{i}\right\}_{i \in \mathrm{X}}$.

By hypothesis $\left\{\Psi_{i}\right\}_{i \in \mathrm{X}}$ is a refinement of $\Omega$, namely

$$
\left.\mid \Omega)_{\mathrm{AB}}=\sum_{i \in \mathrm{X}} \mid \Psi_{i}\right)_{\mathrm{AB}},
$$

and $\Omega \in D_{\rho}$, namely $\left.\left(\left.e\right|_{\mathrm{B}} \mid \Omega\right)_{\mathrm{AB}}=\mid \rho\right)_{\mathrm{A}}$. Accordingly, marginalising both sides of Eq. (37) one has that for every $\left.i \in \mathrm{X},\left(\left.e\right|_{\mathrm{B}} \mid \Psi_{i}\right)_{\mathrm{AB}}=\mid \sigma_{i}\right)_{\mathrm{A}} \in \operatorname{Ref}_{\rho}$. Since $\Psi \in\left\{\Psi_{i}\right\}_{i \in \mathrm{X}}$, this concludes the proof. 